\title{
HOMOLOGY STABILITY FOR ORTHOGONAL GROUPS OVER ALGEBRAICALLY CLOSED FIELDS
}

\author{
By JEAN-LOUIS CATHELINEAU
}

\begin{abstract}
We give the best ranges of stability, for homology of orthogonal groups and special orthogonal groups, over an algebraically closed field, of characteristic different from 2. This answers affirmatively a conjecture asserted in a previous paper. We find distinct ranges of stability for orthogonal and special orthogonal groups, and Milnor $K$-theory appears as an obstruction to stability for special orthogonal groups. The main results are formulated, more generally, for infinite Pythagorean fields.
\end{abstract}

(c) 2007 Elsevier Masson SAS

RÉSUMÉ. - On donne les meilleures bornes de stabilité, pour l'homologie des groupes orthogonaux et spéciaux orthogonaux, sur un corps algébriquement clos de caractéristique différente de 2 . Cela résout par l'affirmative une conjecture antérieure. Les bornes sont distinctes pour les deux types de groupes, et la $K$-théorie de Milnor apparaît comme première obstruction à la stabilité, dans le cas des groupes spéciaux orthogonaux. Les résultats principaux ont plus généralement pour cadre naturel celui où le corps est pythagoricien infini et de caractéristique différente de 2 .

(c) 2007 Elsevier Masson SAS

\section{Introduction}

\subsection{Notations and basic definitions}

For $\mathbf{k}$ a field of characteristic different from 2, let $q_{n}(x)=\sum_{i=1}^{n} x_{i}^{2}$ be the Euclidean quadratic form on $\mathbf{k}^{n}$. We denote by $O(n, \mathbf{k})$ the corresponding orthogonal group, that is the group of orthogonal matrices with coefficients in $\mathbf{k}$. The special orthogonal group is written $S O(n, \mathbf{k})$. The quadric: $Q_{n}(\mathbf{k})=\left\{x \in \mathbf{k}^{n}: q_{n}(x)=1\right\}$ plays a crucial role in what follows.

If $M$ is an $O(n, \mathbf{k})$-module, $M^{t}$ means the same module twisted by the action of the determinant.

Sometimes, when we want to stress the fact that $x \in \mathbf{k}^{n}$ is a vector, we write $\vec{x}$ instead of $x$. We use the notation $\overrightarrow{x y}$, for $y-x$. If $V$ is an affine subspace of $\mathbf{k}^{n}$, the direction of $V$ is the linear space $\vec{V}=\{\vec{x} y: x, y \in V\}$. If $V$ is an affine subspace not containing the origin $0, \bar{V}$ is the linear span of $V$.

A Euclidean space is a quadratic space which is isometric to some $\left(\mathbf{k}^{n}, q_{n}\right)$. A Pythagorean field is a field where the sum of two squares is a square [25]. Pythagorean fields form a natural class containing algebraically closed fields and the real field. From a geometrical point of view, their best characterization is the following: a field is Pythagorean iff every non-degenerate linear subspace of a Euclidean space is Euclidean.

For the rest of the paper, $\mathbf{k}$ is always an infinite field of characteristic $\neq 2$ which is assumed to be Pythagorean, unless specifically stated. This is a rather drastic restriction which discards 
arithmetic difficulties, but it is very unlikely that, without this hypothesis, our results remain true: see Remark 4.6, and the paper [40] for a study in a wider context.

\subsection{Main results}

Homology stability, for linear groups over rings, was a current object of study some years ago (see the references at the end of the paper). One motivation was in Algebraic $K$-theory. The special case of the orthogonal groups for Euclidean forms was considered in Vogtman [40] and Sah [33]. For recent works on homology stability, see also [12,28].

In this paper, we want to return to the case of orthogonal groups of Euclidean forms, for which the subject of Scissors Congruences or extended Hilbert's third problem, has opened new perspectives $[34,15]$. In a series of beautiful papers, the homology of classical Lie groups considered as discrete groups was used by Dupont and Sah, in the study of Scissors Congruences. Goncharov in his article on the volume of hyperbolic manifolds and mixed Tate motives [20], gave another extension of the third problem: it appeared that the homology of orthogonal groups over algebraically closed fields, although not explicit in his work, was indeed relevant (see $[7,8]$ ).

Our purpose here is to give the best ranges of stability for the homology of orthogonal and special orthogonal groups, for Pythagorean fields. Our main results were conjectured in [10], as a natural counterpart to analogous facts on the Lie algebra homology of skew-symmetric matrices.

Recall that we have a natural morphism

$$
H_{i}(O(n, \mathbf{k}), \mathbb{Z}) \rightarrow H_{i}(O(n+1, \mathbf{k}), \mathbb{Z}),
$$

which is induced by the map

$$
A \mapsto\left(\begin{array}{cc}
A & 0 \\
0 & 1
\end{array}\right),
$$

or any of its conjugates. Concerning the orthogonal group, we have

THEOREM 1.1. - The natural map

$$
H_{i}(O(n, \mathbf{k}), \mathbb{Z}) \rightarrow H_{i}(O(n+1, \mathbf{k}), \mathbb{Z}),
$$

is surjective for $n=i$, and bijective for $n \geqslant i+1$.

This theorem is proved in Section 5. It gives the best range of stability. For example, the map

$$
H_{2}(O(2, \mathbb{C}), \mathbb{Z}) \rightarrow H_{2}(O(3, \mathbb{C}), \mathbb{Z})
$$

is surjective but not injective.

For $\mathbf{k}=\mathbb{R}$, Theorem 1.1 is the stability Theorem 1.3 in Sah [33]. One crucial step, in Sah's proof of his theorem, is the existence of circumcenters ${ }^{1}$ for spherical simplices. Let $S t(n, \mathbb{R})$ be the classical Steinberg module of $\mathbb{R}^{n}$ [14], considered as an $O(n, \mathbb{R})$-module. It is implicit, in the work of Sah, that the existence of circumcenters implies the triviality of the homology group $H_{0}(O(n, \mathbb{R}), S t(n, \mathbb{R}))$.

As a main step to the proof of Theorem 1.1, we will generalize this fact in the following form. For the quadratic space $\left(\mathbf{k}^{n}, q_{n}\right)$, we consider the "Tits building" of flags of non-degenerate subspaces (see [7] and Section 4). There is yet a Steinberg $O(n, \mathbf{k})$-module, written $\operatorname{St}\left(q_{n}\right)$, which coincides ${ }^{2}$ with the previous one when $\mathbf{k}=\mathbb{R}$.

\footnotetext{
${ }^{1}$ Circumcenters in hyperbolic geometry, have also been used in [15,6], to prove homological results.

${ }^{2}$ Of course, it is no more a $G L(n, \mathbf{k})$-module in general.

$4^{\mathrm{e}}$ SÉRIE - TOME $40-2007-\mathrm{N}^{\circ} 3$
} 
THEOREM 1.2. - For an infinite Pythagorean field of characteristic not 2, the homology group $H_{0}\left(O(n, \mathbf{k}), S t\left(q_{n}\right)\right)$ is trivial, for $n \geqslant 2$.

The proof of this theorem is completed in Section 4, where we prove also that $H_{0}(O(2, \mathbf{k})$, $\left.S t\left(q_{2}\right)\right) \neq 0$, if $\mathbf{k}$ is not Pythagorean.

By contrast, for the twisted module $S t\left(q_{n}\right)^{t}$ where $n$ is an even integer, the homology group $H_{0}\left(O(n, \mathbf{k}), S t\left(q_{n}\right)^{t}\right)$ which is a so-called scissors congruence group [7,8] seems to be highly non-trivial in general.

If one tries to generalize the circumcenter argument of Sah, one is rapidly confronted with the annoying fact that Geometry, in a Euclidean space over a Pythagorean field, is more complicated than in the case of the real field. The main reason is the presence of degenerate subspaces. In Sections 2 and 3, we introduce and study the necessary material for avoiding this difficulty. Crucial arguments use elementary algebraic geometry.

Here is our main result concerning stability for special orthogonal groups. The different ranges of stability between orthogonal and special orthogonal groups are related to the fact that orthogonal groups are not connected as algebraic groups [4].

THEOREM 1.3. - The natural map

$$
H_{i}(S O(n, \mathbf{k}), \mathbb{Z}[1 / 2]) \rightarrow H_{i}(S O(n+1, \mathbf{k}), \mathbb{Z}[1 / 2]),
$$

is surjective for $n=2 i$, and bijective for $n \geqslant 2 i+1$.

The kernel of the map

$$
H_{n}(S O(2 n, \mathbf{k}), \mathbb{Z}[1 / 2]) \rightarrow H_{n}(S O(2 n+1, \mathbf{k}), \mathbb{Z}[1 / 2]),
$$

is isomorphic to the homology group $H_{n}\left(O(2 n, \mathbf{k}), \mathbb{Z}[1 / 2]^{t}\right)$, with coefficients in the determinant module $\mathbb{Z}[1 / 2]^{t}$.

A special case of this theorem for $\mathbf{k}=\mathbb{R}$ and homology in degree 2 , but with coefficients in $\mathbb{Z}$, is also found in Sah [33].

To prove this theorem, we generalize slightly in Section 6 the results of [6,7]. Recall that the involution of $H_{i}(S O(n, \mathbf{k}), \mathbb{Z}[1 / 2])$, induced by the action by conjugation of $O(n, \mathbf{k})$ in $S O(n, \mathbf{k})$, is responsible for a decomposition into eigenspaces

$$
H_{i}(S O(n, \mathbf{k}), \mathbb{Z}[1 / 2])=H_{i}(S O(n, \mathbf{k}), \mathbb{Z}[1 / 2])^{+} \oplus H_{i}(S O(n, \mathbf{k}), \mathbb{Z}[1 / 2])^{-} .
$$

This decomposition is related to the homology groups of $O(n, \mathbf{k})$ as follows. To the extension

$$
0 \rightarrow S O(n, \mathbf{k}) \rightarrow O(n, \mathbf{k}) \rightarrow\{ \pm 1\} \rightarrow 0
$$

are associated two Hochschild-Serre spectral sequences, with coefficients in $\mathbb{Z}[1 / 2]$ and $\mathbb{Z}[1 / 2]^{t}$. From the collapsing of these spectral sequences, we get

$$
H_{i}(S O(n, \mathbf{k}), \mathbb{Z}[1 / 2])^{+} \cong H_{i}(O(n, \mathbf{k}), \mathbb{Z}[1 / 2])
$$

and

$$
H_{i}(S O(n, \mathbf{k}), \mathbb{Z}[1 / 2])^{-} \cong H_{i}\left(O(n, \mathbf{k}), \mathbb{Z}[1 / 2]^{t}\right) .
$$

Concerning these twisted homology groups, we find that 
THEOREM 1.4. - (i) For $i \geqslant 0, H_{i}\left(O(2 n+1, \mathbf{k}), \mathbb{Z}[1 / 2]^{t}\right)=0$.

(ii) For $i<n, H_{i}\left(O(2 n, \mathbf{k}), \mathbb{Z}[1 / 2]^{t}\right)=0$.

In particular: $H_{i}(O(2 n, \mathbf{k}), \mathbb{Z}[1 / 2])=H_{i}(S O(2 n, \mathbf{k}), \mathbb{Z}[1 / 2])$, for $n>i$.

Theorem 1.3 is a direct consequence of Theorems 1.1 and 1.4.

The proof of Part (i) in Theorem 1.4 is a simple consequence of the "center kills" lemma (see for example [15], Lemma 5.4). Indeed for $n$ odd, $-\mathrm{Id}$ is in the center of $O(n)$ and acts by -1 in $\mathbb{Z}[1 / 2]^{t}$, which is 2 -divisible.

For coefficients in $\mathbb{Q}$, Theorem 1.4(ii) is a special case of a result of [6,7]. We will rework the proof, for coefficient in $\mathbb{Z}[1 / 2]$, in Section 6.

In Section 6.3 we address the special case of quadratically closed fields ${ }^{3}$.

Let $K_{n}^{M}(\mathbf{k})$ denote the Milnor $K$-theory of $\mathbf{k}$ [27]. For a quadratically closed field, it is known that $K_{n}^{M}(\mathbf{k})$ is uniquely 2-divisible, for $n \geqslant 2$, that is

$$
K_{n}^{M}(\mathbf{k}) \otimes \mathbb{Z}[1 / 2]=K_{n}^{M}(\mathbf{k}), \quad \text { for } n \geqslant 2 .
$$

For $n=0,1$

$$
\begin{aligned}
& K_{0}^{M}(\mathbf{k}) \otimes \mathbb{Z}[1 / 2]=\mathbb{Z}[1 / 2], \\
& K_{1}^{M}(\mathbf{k}) \otimes \mathbb{Z}[1 / 2]=\mathbf{k}^{\times} / \boldsymbol{\mu}_{2}(\mathbf{k}),
\end{aligned}
$$

where $\mathbf{k}^{\times}$is the multiplicative group of $\mathbf{k}$, and $\boldsymbol{\mu}_{2}(\mathbf{k})$ is the 2-primary part of the group of roots of unity.

We can now make precise the obstruction to stability for $S O(n, \mathbf{k})$.

THEOREM 1.5. - If $\mathbf{k}$ is quadratically closed

$$
H_{n}(S O(2 n, \mathbf{k}), \mathbb{Z}[1 / 2])^{-} \cong H_{n}\left(O(2 n, \mathbf{k}), \mathbb{Z}[1 / 2]^{t}\right) \cong K_{n}^{M}(\mathbf{k}) \otimes \mathbb{Z}[1 / 2] .
$$

We end the paper in Section 6.4, by a complement in the case of the real field.

\subsection{Applications and complements}

If $\mathbf{k}$ is quadratically closed, the orthogonal group $O(2 n, \mathbf{k})$ is isomorphic to the orthogonal group $O(n, n, \mathbf{k})$ of the hyperbolic quadratic form: $x_{1} x_{n+1}+\cdots+x_{n} x_{2 n}$. In this special case, we can relate our results to Suslin's stability theorem for $G L(n, \mathbf{k})$ (see Suslin [36], and the remarks at the end of the introduction in [8]). Identifying $O(2 n, \mathbf{k})$ to $O(n, n, \mathbf{k})$, the hyperbolic morphism

$$
\begin{aligned}
& G L(n, \mathbf{k}) \rightarrow O(n, n, \mathbf{k}), \\
& M \mapsto\left(\begin{array}{cc}
M & 0 \\
0 & { }^{t} M^{-1}
\end{array}\right)
\end{aligned}
$$

induces a composed morphism

$$
H_{i}(G L(n, \mathbf{k}), \mathbb{Z}[1 / 2]) \rightarrow H_{i}(S O(2 n, \mathbf{k}), \mathbb{Z}[1 / 2]) \rightarrow H_{i}\left(O(2 n, \mathbf{k}), \mathbb{Z}[1 / 2]^{t}\right) .
$$

The maps

$$
H_{i}\left(O(2 m, \mathbf{k}), \mathbb{Z}[1 / 2]^{t}\right) \rightarrow H_{i}\left(O(2 m+2, \mathbf{k}), \mathbb{Z}[1 / 2]^{t}\right),
$$

\footnotetext{
${ }^{3}$ That is fields where each element is a square. Such fields are exactly Pythagorean fields where -1 is a square [25].

$4^{\mathrm{e}}$ SÉRIE - TOME $40-2007-\mathrm{N}^{\circ} 3$
} 
are trivial, since the groups $H_{i}\left(O(2 m+1, \mathbf{k}), \mathbb{Z}[1 / 2]^{t}\right)$ are zero. We get by naturality a morphism

$$
\frac{H_{i}(G L(n, \mathbf{k}), \mathbb{Z}[1 / 2])}{\operatorname{Im} H_{i}(G L(n-1, \mathbf{k}), \mathbb{Z}[1 / 2])} \longrightarrow H_{i}\left(O(2 n, \mathbf{k}), \mathbb{Z}[1 / 2]^{t}\right)
$$

where $\operatorname{Im} H_{i}(G L(n-1, \mathbf{k}), \mathbb{Z}[1 / 2])$, is the image of the stabilization map.

PROPOSITION 1.6. - For a quadratically closed field, the previous morphism

$$
\frac{H_{i}(G L(n, \mathbf{k}), \mathbb{Z}[1 / 2])}{\operatorname{Im} H_{i}(G L(n-1, \mathbf{k}), \mathbb{Z}[1 / 2])} \longrightarrow H_{i}\left(O(2 n, \mathbf{k}), \mathbb{Z}[1 / 2]^{t}\right),
$$

is an isomorphism, for $i \leqslant n$. Actually these groups are identified to $K_{n}^{M}(\mathbf{k}) \otimes \mathbb{Z}[1 / 2]$, for $i=n$, and are reduced to 0 , for $i<n$.

Concerning the composed morphism

$$
H_{i}(G L(n, \mathbf{k}), \mathbb{Z}[1 / 2]) \rightarrow H_{i}(S O(2 n, \mathbf{k}), \mathbb{Z}[1 / 2]) \rightarrow H_{i}(O(2 n, \mathbf{k}), \mathbb{Z}[1 / 2]),
$$

we have also the following.

PROPOSITION 1.7. - For a quadratically closed field, the morphism induced by the hyperbolic map

$$
H_{i}(G L(n, \mathbf{k}), \mathbb{Z}[1 / 2]) \rightarrow H_{i}(O(2 n, \mathbf{k}), \mathbb{Z}[1 / 2]),
$$

is surjective for $n \geqslant i$.

For a quadratically closed field, the Witt ring is $\mathbb{Z} / 2 \mathbb{Z}$. The result then follows from Karoubi [23], Théorème 3.5, Suslin's stability theorem, and our own stability results.

In Friedlander [18], one finds the following theorem

THEOREM. - Let $\overline{\mathbb{F}}_{p}$ be the algebraic closure of the finite field $\mathbb{F}_{p}$, with $p$ an odd prime; the natural map

$$
H_{i}\left(S O\left(n, \overline{\mathbb{F}}_{p}\right), \mathbb{Z}\right) \rightarrow H_{i}\left(S O\left(n+1, \overline{\mathbb{F}}_{p}\right), \mathbb{Z}\right),
$$

is bijective for $n \geqslant i+2$.

Combined with our own results, this has the following consequence

PROPOSITION 1.8. - If $\overline{\mathbb{F}}_{p}$ is the algebraic closure of $\mathbb{F}_{p}(p \neq 2)$,

$$
H_{i}\left(O\left(n, \overline{\mathbb{F}}_{p}\right), \mathbb{Z}[1 / 2]^{t}\right)=0, \quad \text { for } n \geqslant i+2 .
$$

There is no contradiction with Theorem 1.5 because $K_{n}^{M}\left(\overline{\mathbb{F}}_{p}\right)=0$, for $n \geqslant 2$. Note that the range of stability, in the result of Friedlander, is the best possible. For example, the map

$$
H_{1}\left(S O\left(2, \overline{\mathbb{F}}_{p}\right), \mathbb{Z}\right) \rightarrow H_{1}\left(S O\left(3, \overline{\mathbb{F}}_{p}\right), \mathbb{Z}\right)
$$

is the map: $\overline{\mathbb{F}}_{p}^{\times} \rightarrow 0$, since $S O\left(3, \overline{\mathbb{F}}_{p}\right)$ is equal to its commutator subgroup [13].

Here is another complement, without details. The generalization to orthogonal groups, over rings of dual numbers $\mathbf{k}[X] /\left(X^{2}\right)$, along the ideas of [6] is easy. If moreover $\mathbf{k}$ has characteristic 0, we can apply [6] Proposition 1 to get the following corollary of our main results. 
THEOREM 1.9. - For $\mathbf{k}$ a Pythagorean field of characteristic 0 , let so $(n, \mathbf{k})$ be the Lie algebra of skew-symmetric $(n \times n)$-matrices. We consider so $(n, \mathbf{k})$ as an $O(n, \mathbf{k})$-module, by the adjoint action, and write $\operatorname{so}(n, \mathbf{k})^{t}$ the adjoint action twisted by the determinant.

(i) The natural map

$$
H_{i}(O(n, \mathbf{k}), s o(n, \mathbf{k})) \rightarrow H_{i}(O(n+1, \mathbf{k}), s o(n+1, \mathbf{k})),
$$

is surjective for $n=i+1$, and bijective for $n \geqslant i+2$.

(ii) The natural map

$$
H_{i}(S O(n, \mathbf{k}), s o(n, \mathbf{k})) \rightarrow H_{i}(S O(n+1, \mathbf{k}), s o(n+1, \mathbf{k})),
$$

is surjective for $n=2 i+1$, and bijective for $n \geqslant 2 i+2$.

(iii) For $i \geqslant 0, H_{i}\left(O(2 n+1, \mathbf{k})\right.$, so $\left.(2 n+1, \mathbf{k})^{t}\right)=0$.

For $i<n-1, H_{i}\left(O(2 n, \mathbf{k}), s o(2 n, \mathbf{k})^{t}\right)=0$.

For $n>i+1, H_{i}(O(2 n, \mathbf{k}), s o(n, \mathbf{k}))=H_{i}(S O(2 n, \mathbf{k}), s o(n, \mathbf{k}))$.

(iv) If moreover -1 is a square,

$$
H_{n-1}\left(O(2 n, \mathbf{k}), s o(2 n, \mathbf{k})^{t}\right) \cong \Omega_{\mathbf{k}}^{n-1},
$$

where $\Omega_{\mathbf{k}}^{n}$ is the space of absolute Kähler differentials of degree $n$.

\subsection{Problems}

Here is a list of open questions.

(A) What can be said of Theorem 1.3, considering coefficients in $\mathbb{Z}$, instead of $\mathbb{Z}[1 / 2]$ ? Note that the result is yet true for $i=2$, as in [33] for the case of $\mathbf{k}=\mathbb{R}$.

(B) What could be the kernel of the map: $H_{n}(O(n, \mathbf{k}), \mathbb{Z}) \rightarrow H_{n}(O(n+1, \mathbf{k}), \mathbb{Z})$ ? Even in the simpler analogous case of the Lie algebra homology of skew-symmetric matrices, the corresponding kernel is not known. It should be related to the invariant theory of matrices (see $[26,10])$.

(C) What are the homology groups $H_{i}\left(O(2 n, \mathbf{k}), \mathbb{Z}^{t}\right)$, for $n<i<2 n$ ? These homology groups are potentially interesting, in the study of scissors congruences. They could be related to Adams decompositions, in algebraic $K$-theory.

(D) One can define, as above, a Steinberg $O(E)$-module $S t(E)$, for any non-degenerate quadratic space $(E, q)$ (see [9]). Calculate $H_{0}(O(E), S t(E)$ ).

(E) Generalize our stability results to a good class of local rings, with infinite Pythagorean residue fields.

(F) Make precise the ranges of homology stability for orthogonal groups of Euclidean forms over non-Pythagorean fields (see [40]).

(G) What is the relation if any, between Milnor $K$-theory and homology stability problems for orthogonal groups over rings (see $[29,19]$ for $G L_{n}$ )?

\subsection{Simplified notations}

Henceforth we will write $O(n)$ (resp. $S O(n), S t(n))$, instead of $O(n, \mathbf{k})$ (resp. $S O(n, \mathbf{k})$, $\left.\operatorname{St}\left(q_{n}\right)\right)$.

For the convenience of the reader, and also to be as self contained as possible, I have resumed, along the text, several arguments contained in my previous papers on orthogonal groups.

4e SÉRIE - TOME $40-2007-\mathrm{N}^{\circ} 3$ 


\section{Configurations of points and lines}

\subsection{Simplices}

We will consider two types of simplices: $p$-simplices of vectors: $\left(v_{0}, v_{1}, \ldots, v_{p}\right)$, with $v_{i} \in \mathbf{k}^{n}$, and $p$-simplices of linear subspaces of $\mathbf{k}^{n}$ of dimension $1:\left(L_{0}, L_{1}, \ldots, L_{p}\right)$, called simplices of lines. These lines will be also considered as points of the projective space $\mathbb{P}\left(\mathbf{k}^{n}\right)$.

The rank: $\mathrm{rk} \omega$, of a simplex $\omega=\left(X_{0}, X_{1}, \ldots, X_{p}\right)$, is the dimension of the linear space generated by its vertices $X_{i}$. For $I=\left\{i_{0}<i_{1}<\cdots<i_{r}\right\} \subset\{0,1, \ldots, p\}$, the related face of $\left(X_{0}, X_{1}, \ldots, X_{p}\right)$ is $\left(X_{i_{0}}, X_{i_{1}}, \ldots, X_{i_{p}}\right)$.

Definition 2.1. - A $p$-simplex $\omega$ is generic, if $\operatorname{rk} \omega=p+1$. Note that if $\left(v_{0}, v_{1}, \ldots, v_{p}\right)$ is a generic simplex of vectors of $\mathbf{k}^{n}$, the affine subspace generated by the points $\left\{v_{0}, v_{1}, \ldots, v_{p}\right\}$ does not contain the origin.

A $p$-simplex $\omega$ is non-degenerate, if the linear space generated by its vertices is nondegenerate.

A $p$-simplex $\omega$ is geometric, if all its faces are non-degenerate.

A $p$-simplex $\left(v_{0}, v_{1}, \ldots, v_{p}\right)$, with $v_{i} \in Q_{n}(\mathbf{k})$, is said to be strongly geometric, if it is generic and geometric, and if for each subset $I$ of $\{0,1, \ldots, p\}$ the vector space $\operatorname{Vect}\left\langle\overrightarrow{v_{i} v_{j}}, i, j \in I\right\rangle$ is non-degenerate.

We now introduce a few notations specific to a strongly geometric simplex $\omega=\left(v_{0}, v_{1}, \ldots, v_{p}\right)$. For $I \subset\{0,1, \ldots, p\}$, the vector space: $\operatorname{Vec}\left\langle\overrightarrow{v_{i} v_{j}}, i, j \in I\right\rangle$ is a non-degenerate subspace of codimension 1 , in the non-degenerate vector space $\operatorname{Vec}\left\langle\overrightarrow{v_{i}}, i \in I\right\rangle$. We denote by $L_{I}$ the orthogonal of $\operatorname{Vec}\left\langle\overrightarrow{v_{i} v_{j}}, i, j \in I\right\rangle$ in $\operatorname{Vec}\left\langle v_{i}, i \in I\right\rangle$. For $I$ reduced to one element $i, L_{I}=\overrightarrow{\mathbf{k} v_{i}}$. For $i \in\{0,1, \ldots, p\}$, and any permutation $\sigma$ of $\{0,1, \ldots, p\}$, we put $L_{i}^{\sigma}:=L_{\{\sigma(0), \sigma(1), \ldots, \sigma(i)\}}$. We remark that the simplex of lines $\left(L_{0}^{\sigma}, L_{1}^{\sigma}, \ldots, L_{p}^{\sigma}\right)$ can be non-generic and is not geometric in general (nevertheless one can check that: for $0 \leqslant r \leqslant s \leqslant p$, the face $\left(L_{r}^{\sigma}, L_{r+1}^{\sigma}, \ldots, L_{s}^{\sigma}\right)$ is nondegenerate).

The following proposition will be used in Section 3.

PROPOSITION 2.2. - Let $\omega=\left(v_{0}, v_{1}, \ldots, v_{p}\right)$ be a strongly geometric simplex, $\sigma$ a permutation of $\{0,1, \ldots, p\}$, and $\tau$ the transposition (01). Denote by $H$ the non-degenerate linear subspace of codimension 1 of $\mathbf{k}^{n}$, orthogonal to $\overrightarrow{v_{\sigma(0)} v_{\sigma(1)}}$, and let $s_{H}$ be the orthogonal symmetry around $H$. We have

$$
s_{H}\left(\left(L_{0}^{\sigma}, L_{1}^{\sigma}, \ldots, L_{p}^{\sigma}\right)\right)=\left(L_{0}^{\sigma \tau}, L_{1}^{\sigma \tau}, \ldots, L_{p}^{\sigma \tau}\right) .
$$

As a result, these two simplices are generic and geometric or not, at the same time.

Since $L_{0}^{\sigma}=\mathbf{k} \overrightarrow{v_{\sigma(0)}}$, and $L_{0}^{\sigma \tau}=\mathbf{k} \overrightarrow{v_{\sigma(1)}}$, we have $s_{H}\left(L_{0}^{\sigma}\right)=L_{0}^{\sigma \tau}$. On the other hand, $L_{i}^{\sigma}=L_{i}^{\sigma \tau}$, for $i \geqslant 1$, and these lines are in the hyperplane $H$.

DEFINITION 2.3. - A strongly geometric simplex $\omega=\left(v_{0}, v_{1}, \ldots, v_{p}\right)$ is said to be nice if, for all $\sigma$, the $p$-simplex of lines $\left(L_{0}^{\sigma}, L_{1}^{\sigma}, \ldots, L_{p}^{\sigma}\right)$ is generic and geometric. A geometric simplex $\omega=\left(v_{0}, v_{1}, \ldots, v_{p}\right)$, with $v_{i} \in Q_{n}(\mathbf{k})$, will be called excellent, if all its generic faces are nice.

Remark 2.4. - A 0-simplex $\left(v_{0}\right)$, with $q_{n}\left(v_{0}\right)=1$, is nice, as well as a strongly geometric 1 -simplex $\left(v_{0}, v_{1}\right)$. The faces of a nice simplex are nice, and the faces of an excellent simplex are excellent. 


\subsection{Complexes of configurations}

We introduce three complexes of $O(n)$-modules:

The projective complex $\left(B_{*}(n), d\right)$, where $B_{p}(n)$ is the free abelian group generated by the geometric $p$-simplices of lines $\left(L_{0}, L_{1}, \ldots, L_{p}\right)$, with the standard differential

$$
d\left(\left(L_{0}, L_{1}, \ldots, L_{p}\right)\right)=\sum_{i=0}^{p}(-1)^{i}\left(L_{0}, \ldots, \widehat{L_{i}}, \ldots, L_{p}\right) .
$$

The geometric complex of $Q_{n}(\mathbf{k}):\left(C_{*}(n), d\right)$. Here $C_{p}(n)$ is the free abelian group generated by the geometric $p$-simplices $\left(v_{0}, v_{1}, \ldots, v_{p}\right)$, with $v_{i} \in Q_{n}(\mathbf{k})$, and the differential is analogous to the previous one.

The excellent complex $\left(D_{*}(n), d\right)$ is the subcomplex of $\left(C_{*}(n), d\right)$, generated by the excellent simplices.

These complexes are naturally $\mathbb{Z}$-augmentated, with augmentations $\epsilon_{B}, \epsilon_{C}, \epsilon_{D}:(X) \mapsto 1$. The map which associates to a vector $\vec{v}$ the line $\mathbf{k} \vec{v}$ induces a morphism of complexes

$$
\Psi: C_{*}(n) \rightarrow B_{*}(n) .
$$

This morphism is surjective, when $\mathbf{k}$ is Pythagorean.

The following theorem is a crucial step.

THEOREM 2.5. - The three augmented complexes

$$
\left(B_{*}(n), d, \epsilon_{B}\right),\left(C_{*}(n), d, \epsilon_{C}\right),\left(D_{*}(n), d, \epsilon_{D}\right),
$$

are acyclic, for $n \geqslant 1$.

This result is a consequence of the following extension property, which is proved in the next section. One can check directly that the augmentations identify the 0-homology groups of these complexes to $\mathbb{Z}$. In the case of $D_{*}(n)$, one remarks that if $v_{1}, v_{2} \in Q_{n}(\mathbf{k})$, one can find $w \in Q_{n}(\mathbf{k})$, such that $\overrightarrow{w v_{1}}$ and $\overrightarrow{w v_{2}}$ are anisotropic.

Proposition 2.6. - (i) For a non-degenerate simplex of lines $\left(L_{0}, L_{1}, \ldots, L_{p}\right)$, the set of anisotropic lines $L$, such that $\left(L, L_{0}, L_{1}, \ldots, L_{p}\right)$ is non-degenerate, contains a non-empty Zariski open subset of the projective space $\mathbb{P}\left(\mathbf{k}^{n}\right)$.

(ii) For a non-degenerate simplex $\left(v_{0}, v_{1}, \ldots, v_{p}\right)$ of points of $Q_{n}(\mathbf{k})$, the set of points $v \in Q_{n}(\mathbf{k})$, such that $\left(v, v_{0}, v_{1}, \ldots, v_{p}\right)$ is non-degenerate, contains a non-empty Zariski open subset of $Q_{n}(\mathbf{k})$.

(iii) For a nice simplex $\left(v_{0}, v_{1}, \ldots, v_{p}\right)$ of rank $<n$, the set of points $v \in Q_{n}(\mathbf{k})$, such that $\left(v, v_{0}, v_{1}, \ldots, v_{p}\right)$ is nice, is exactly a non-empty Zariski open subset of $Q_{n}(\mathbf{k})$.

We deduce Theorem 2.5 from the extension property. For this, we need a lemma. Fix an algebraic closure $\overline{\mathbf{k}}$ of $\mathbf{k}$, and let $Q_{n}(\overline{\mathbf{k}})=\left\{x \in \overline{\mathbf{k}}: q_{n}(x)=1\right\}$. The following result, true for any infinite field, will be used repeatedly to prove the existence of points satisfying a finite set of algebraic requirements.

LEMma 2.7. - The set $Q_{n}(\mathbf{k})$ is dense in $Q_{n}(\overline{\mathbf{k}})$ for the Zariski topology. Since $Q_{n}(\overline{\mathbf{k}})$ is irreducible, this implies that two non-empty open sets of $Q_{n}(\mathbf{k})$ have a non-empty intersection. The same is true for the projective space.

$4^{\text {e }}$ SÉRIE - TOME $40-2007-\mathrm{N}^{\circ} 3$ 
Of course the property holds for the affine space, since $\mathbf{k}$ is infinite. The proof of the lemma is an easy consequence of this, using the irreducibility of $Q_{n}(\overline{\mathbf{k}})$, and the stereographic projection from the point $(-1,0, \ldots, 0)$

$$
\left(Q_{n}(\overline{\mathbf{k}}) \backslash\left\{\left(-1, x_{2}, \ldots, x_{n}\right): \sum_{i=2}^{n} x_{i}^{2}=0\right\}\right) \longrightarrow\left(\overline{\mathbf{k}}^{n-1} \backslash\left\{\left(0, x_{2}, \ldots, x_{n}\right): \sum_{i=2}^{n} x_{i}^{2}=-1\right\}\right) .
$$

In the same vein, the result works for the projective space. More generally, it is well known that the lemma is true for any "rational variety" defined over an infinite field.

From the previous lemma and Proposition 2.6, we deduce that for a geometric simplex $\left(v_{0}, v_{1}, \ldots, v_{p}\right)$ (resp. an excellent simplex), the set of $v \in Q_{n}(\mathbf{k})$, such that $\left(v, v_{0}, v_{1}, \ldots, v_{p}\right)$ is geometric (resp. excellent), contains a non-empty Zariski open subset of $Q_{n}(\mathbf{k})$. In the same way, for a geometric simplex $\left(L_{0}, L_{1}, \ldots, L_{p}\right)$, the set of $L \in \mathbb{P}\left(\mathbf{k}^{n}\right)$, such that $\left(L, L_{0}, L_{1}, \ldots, L_{p}\right)$ is geometric, contains a non-empty Zariski open subset of $\mathbb{P}\left(\mathbf{k}^{n}\right)$. Then let $\omega=\sum_{i} \pm\left(X_{0}^{i}, X_{1}^{i}, \ldots, X_{p}^{i}\right)$ be a cycle in one of the three complexes $C_{*}(n), D_{*}(n)$ and $B_{*}(n)$. By the Zariski argument, it is clear now that there exists an $X$ such that for all $i,\left(X, X_{0}^{i}, X_{1}^{i}, \ldots, X_{p}^{i}\right)$ is yet a simplex of the corresponding complex. For such an $X$, $d(X \star \omega)=\omega$, where $X \star \omega:=\sum_{i} \pm\left(X, X_{0}^{i}, X_{1}^{i}, \ldots, X_{p}^{i}\right)$ is the join of $X$ with $\omega$. This proves Theorem 2.5.

We conclude this section with a corollary of Theorem 2.5. Let $C_{*}^{\prime}(n)\left(\right.$ resp. $\left.B_{*}^{\prime}(n), D_{*}^{\prime}(n)\right)$ be the subcomplex of $C_{*}(n)$ (resp. $B_{*}(n), D_{*}(n)$ ), generated by the simplices of rank $<n$. We have the quotient complexes

$$
\begin{aligned}
& \hat{C}_{*}(n):=C_{*}(n) / C_{*}^{\prime}(n), \\
& \hat{B}_{*}(n):=B_{*}(n) / B_{*}^{\prime}(n),
\end{aligned}
$$

and

$$
\hat{D}_{*}(n):=D_{*}(n) / D_{*}^{\prime}(n) .
$$

COROLLARY 2.8. - For $i<n-2$,

$$
\tilde{H}_{i}\left(C_{*}^{\prime}(n)\right)=H_{i+1}\left(\hat{C}_{*}(n)\right)=0 .
$$

The same is true for the other two complexes $B_{*}(n)$ and $D_{*}(n)$.

Since $\hat{C}_{i}(n)=0$, for $i \leqslant n-2$, this is a consequence of Theorem 2.5, applied to the long homology sequence of the short exact sequence

$$
0 \rightarrow C_{*}^{\prime}(n) \rightarrow C_{*}(n) \rightarrow \hat{C}_{*}(n) \rightarrow 0 .
$$

Remark 2.9. - We observe that the $\mathbb{Z}$-module $\hat{D}_{n-1}(n)$ is generated as a free module, by the nice simplices of rank $n$.

\section{The extension property}

The purpose of this section is the proof of Proposition 2.6.

\subsection{Easy cases}

The cases (i) and (ii) of the proposition are easy. 
Let $\left(L_{0}, L_{1}, \ldots, L_{p}\right)$ be a non-degenerate simplex of lines. If $\sum_{i} L_{i}=\mathbf{k}^{n}$, we can extend this simplex by any anisotropic line. If $\sum_{i} L_{i} \neq \mathbf{k}^{n}$, one sees by using Gram determinants ${ }^{4}$, that the set of anisotropic lines $L$ outside $\sum_{i} L_{i}$, such that the simplex $\left(L, L_{0}, L_{1}, \ldots, L_{p}\right)$ is nondegenerate, is a Zariski open subset of the projective space. And this set is not empty, because it contains any anisotropic line which is orthogonal to $\sum_{i} L_{i}$.

Now let $\left(v_{0}, v_{1}, \ldots, v_{p}\right)$ be a non-degenerate simplex of points of $Q_{n}(\mathbf{k})$. It is enough to consider the case $\operatorname{Vec}\left\langle v_{i}, i\right\rangle \neq \mathbf{k}^{n}$. The set of vectors $v \in \mathbf{k}^{n}$, outside $\operatorname{Vec}\left\langle v_{i}, i\right\rangle$, such that the simplex $\left(v, v_{0}, v_{1}, \ldots, v_{p}\right)$ is non-degenerate, is a Zariski open subset of $\mathbf{k}^{n}$. We have to show that this set intersects the quadric $Q_{n}(\mathbf{k})$. But a point, in the intersection, is given by the intersection of $Q_{n}(\mathbf{k})$ with any anisotropic linear space of dimension 1 , orthogonal to $\operatorname{Vec}\left\langle v_{i}, i\right\rangle$ : such a point exists because $\mathbf{k}$ is Pythagorean (actually, proceeding as in Lemma 3.5 below, one can see that this hypothesis is not necessary here).

\subsection{Extension of nice simplices}

Although elementary, this case needs some care. We solve the extension problem, for nice simplices, by reduction to another problem, concerning flags of affine subspaces of $\mathbf{k}^{n}$. The main calculations will concern "orthoschemes". In Euclidean geometry, a simplex of points $\left(z_{0}, z_{1}, \ldots, z_{p}\right)$ is an orthoscheme if the vectors $\overrightarrow{z_{0} z_{1}}, \overrightarrow{z_{1} z_{2}}, \ldots, \overrightarrow{z_{p-1} z_{p}}$ are orthogonal. The analogous notion in spherical geometry was introduced by Schäfli [34]. In classical Euclidean or spherical geometry, it is well known that circumcenters give decompositions of simplices into orthoschemes.

DEFINITION 3.1. - A good affine flag

$$
V_{0} \subset V_{1} \subset \cdots \subset V_{p},
$$

is a flag of affine subspaces of $\mathbf{k}^{n}$, with the following properties.

(1) $p \leqslant n-2$.

(2) The direction $\overrightarrow{V_{i}}$ of the affine space $V_{i}$ is a non-degenerate linear subspace, of dimension $i$.

(3) If $a_{i}$ is the orthogonal projection of the origin 0 upon $V_{i}$, which is well defined by the previous hypothesis, the simplex of vectors $\left(\overrightarrow{a_{0}}, \overrightarrow{a_{1}}, \ldots, \overrightarrow{a_{p}}\right)$ is generic and geometric.

Note that $V_{0}=\left\{a_{0}\right\}$. We call $a_{0}$ the vertex of the flag.

Basic example. Let $\left(v_{0}, v_{1}, \ldots, v_{p}\right)$ be a nice simplex of rank $<n$ (recall that $v_{i} \in Q_{n}(\mathbf{k})$ ), and let $\sigma$ be a permutation of $\{0,1, \ldots, p\}$. If $V_{i}^{\sigma}$ denote the affine subspace of $\mathbf{k}^{n}$, generated by the points $\left\{v_{\sigma(0)}, \ldots, v_{\sigma(i)}\right\}$, the flag $V_{0}^{\sigma} \subset V_{1}^{\sigma} \subset \cdots \subset V_{p}^{\sigma}$ is a good affine flag with vertex $v_{\sigma(0)}$ in $Q_{n}(\mathbf{k})$. Note that the line $L_{i}^{\sigma}$ is generated by the vector $\overrightarrow{a_{i}}$, with the previous notations.

LEMMA 3.2. - Let: $V_{0} \subset V_{1} \subset \cdots \subset V_{p}$, be a good affine flag.

(i) The affine subspace $V_{i}$ does not contain the origin. The set $\left\{a_{0}, \ldots, a_{i}\right\}$ is an affine frame of $V_{i}$.

The vectors

$$
\overrightarrow{a_{0} a_{1}}, \ldots, \overrightarrow{a_{i-1} a_{i}}
$$

form an orthogonal basis of $\vec{V}_{i}$, and the vectors

$$
\overrightarrow{a_{0} a_{1}}, \ldots, \overrightarrow{a_{i-1} a_{i}}, \overrightarrow{a_{i}},
$$

\footnotetext{
${ }^{4}$ Recall that, for a symmetric form $\langle.,$.$\rangle , the Gram determinant \operatorname{Gram}\left(v_{1}, \ldots, v_{l}\right)$ of the vectors: $v_{1}, \ldots, v_{l}$, is the determinant of the matrix: $\left(\left\langle v_{i}, v_{j}\right\rangle\right), i, j \in\{1, \ldots, l\}$.

$4^{\mathrm{e}}$ SÉRIE - TOME $40-2007-\mathrm{N}^{\circ} 3$
} 
form an orthogonal basis of the vector space $\overline{V_{i}}=\mathbf{k} \overrightarrow{a_{0}}+\overrightarrow{V_{i}}$ generated by $V_{i}$.

(ii) We have

$$
\begin{aligned}
& q_{n}\left(\overrightarrow{a_{i}}\right) \neq 0, \quad i=0, \ldots, p, \\
& q_{n}\left(\overrightarrow{a_{i} a_{j}}\right) \neq 0, \quad 0 \leqslant i<j \leqslant p .
\end{aligned}
$$

Only the part (ii) needs a proof. By the hypothesis, for $i<j$, the linear space generated by $\overrightarrow{a_{i}}$ and $\overrightarrow{a_{j}}$ is non-degenerate of dimension 2. But since $\overrightarrow{a_{j}}$ is orthogonal to $\overrightarrow{a_{i} a_{j}}$ for $j>i$, these two vectors are an orthogonal basis of a non-degenerate space.

Definition 3.3. - A vector $\vec{u}$ is said to be admissible for the good affine flag

$$
V_{0} \subset V_{1} \subset \cdots \subset V_{p}
$$

if the following conditions are satisfied.

(1) $\vec{u}$ is not in $\overrightarrow{V_{p}}$.

(2) The vector space $\overrightarrow{W_{i}}=\mathbf{k} \vec{u}+\vec{V}_{i}$ is non-degenerate, for $i \in\{0,1, \ldots, p\}$.

(3) If $b_{i}$ is the orthogonal projection of the origin upon the affine space $W_{i}=a_{0}+$ $\overrightarrow{W_{i}}$, the simplex of vectors $\left(\overrightarrow{a_{0}}, \ldots, \overrightarrow{a_{i}}, \overrightarrow{b_{i}}, \ldots, \overrightarrow{b_{p}}\right)$ is generic and geometric, for any $i \in\{0,1, \ldots, p\}$.

We remark that, Hypothesis (2), for $i=0$, implies that an admissible vector is anisotropic. Also by Hypothesis (3), $\overrightarrow{a_{0}}$ and $\vec{u}$ are not orthogonal.

DEFinition 3.4. - Let $V_{0} \subset V_{1} \subset \cdots \subset V_{p}$, be a good affine flag with vertex $a_{0} \in Q_{n}(\mathbf{k})$. A point $\omega$ of $Q_{n}(\mathbf{k})$ is said to be admissible, if the vector $\overrightarrow{a_{0} \omega}$ is admissible.

LEMMA 3.5. - Let $V_{0} \subset V_{1} \subset \cdots \subset V_{p}$, be a good affine flag with vertex $a_{0} \in Q_{n}(\mathbf{k})$, and $\vec{u}$ an admissible vector. The affine line $a_{0}+\mathbf{k} \vec{u}$ intersects $Q_{n}(\mathbf{k})$ in a second point $\omega \neq a_{0}$ which is admissible.

From the above remark, $q_{n}(\vec{u}) \neq 0$ and $\left\langle\overrightarrow{a_{0}}, \vec{u}\right\rangle \neq 0$. We have

$$
\vec{\omega}=\overrightarrow{a_{0}}-2 \frac{\left\langle\overrightarrow{a_{0}}, \vec{u}\right\rangle}{q_{n}(\vec{u})} \vec{u}
$$

PROPOSITION 3.6. - The set of admissible vectors, for the good affine flag

$$
V_{0} \subset V_{1} \subset \cdots \subset V_{p}
$$

is a non-empty Zariski open cone in $\mathbf{k}^{n}$.

COROLLARY 3.7. - For a good affine flag $V_{0} \subset V_{1} \subset \cdots \subset V_{p}$, with vertex in $Q_{n}(\mathbf{k})$, the set of admissible points is a non-empty Zariski open subset of $Q_{n}(\mathbf{k})$.

The corollary is clear from the previous lemma.

For the proof of the proposition, we start from a good affine flag $V_{0} \subset V_{1} \subset \cdots \subset V_{p}$. The fact that the set of admissible vectors, for this flag, is Zariski open is a standard matter of Gram determinants which we omit. In our case, this involves not only polynomials in the coordinates, but also rational functions coming from solutions of systems of linear equations.

The really crucial point is to show the existence of an admissible vector.

We fix, for the rest of the proof, an anisotropic vector $\vec{w}$ orthogonal to $\overline{V_{p}}$. Such a vector exists because $\operatorname{dim} \overline{V_{p}}<n$, since $p \leqslant n-2$. For $\lambda \in \mathbf{k}^{\times}$, let $\overrightarrow{w_{\lambda}}=\overrightarrow{a_{p}}+\lambda \vec{w}$. The vector $\overrightarrow{w_{\lambda}}$ is 
anisotropic if

$$
\lambda^{2} \neq-\frac{q_{n}\left(\overrightarrow{a_{p}}\right)}{q_{n}(\vec{w})} .
$$

We assume this condition for $\lambda$, in what follows. This implies that the vector spaces

$$
\overrightarrow{W_{i}}=\mathbf{k} \overrightarrow{w_{\lambda}}+\overrightarrow{V_{i}}
$$

are non-degenerate.

We denote yet by $b_{i}$ the orthogonal projection of the origin on the affine space

$$
W_{i}=a_{0}+\overrightarrow{W_{i}}=\mathbf{k} \overrightarrow{w_{\lambda}}+V_{i}
$$

By a series of lemmas, we now prove that, except for a finite set of values of $\lambda$, the vector $\overrightarrow{w_{\lambda}}$ is admissible.

LEMMA 3.8. - (i) The vector $\overrightarrow{a_{i} b_{i}}$ does not depend on $i$, and coincides with the orthogonal projection of $-\overrightarrow{a_{p}}$ on $\mathbf{k} \overrightarrow{w_{\lambda}}$, which is $-\frac{q_{n}\left(\overrightarrow{a_{p}}\right)}{q_{n}\left(\overrightarrow{w_{\lambda}}\right)} \overrightarrow{w_{\lambda}}$. This gives, for $0 \leqslant i \leqslant n$

$$
q_{n}\left(\overrightarrow{a_{i} b_{i}}\right)=\frac{q_{n}\left(\overrightarrow{a_{p}}\right)^{2}}{q_{n}\left(\overrightarrow{w_{\lambda}}\right)} \neq 0
$$

(ii) We have $\overrightarrow{b_{i} b_{j}}=\overrightarrow{a_{i} a_{j}}$. So that, for $0 \leqslant i<j \leqslant p$

$$
q_{n}\left(\overrightarrow{b_{i} b_{j}}\right) \neq 0 \text {. }
$$

For the proof, we remark that the vectors $\overrightarrow{a_{i} b_{i}}$ and $\overrightarrow{w_{\lambda}}$ are collinear, because $\overrightarrow{a_{i} b_{i}}$ is contained in $\overrightarrow{W_{i}}$ and is orthogonal to $\overrightarrow{V_{i}}$. We have $\overrightarrow{a_{i} b_{i}}=\overrightarrow{a_{i} a_{p}}-\overrightarrow{a_{p}}+\overrightarrow{b_{i}}$, and $\overrightarrow{a_{i} a_{p}}$ and $\overrightarrow{b_{i}}$ are orthogonal to $\overrightarrow{w_{\lambda}}$. This implies

$$
\overrightarrow{a_{i} b_{i}}=-\frac{\left\langle\overrightarrow{a_{p}}, \overrightarrow{w_{\lambda}}\right\rangle}{q_{n}\left(\overrightarrow{w_{\lambda}}\right)} \overrightarrow{w_{\lambda}}=-\frac{q_{n}\left(\overrightarrow{a_{p}}\right)}{q_{n}\left(\overrightarrow{w_{\lambda}}\right)} \overrightarrow{w_{\lambda}}
$$

From Part (i), we deduce that

$$
\overrightarrow{b_{i} b_{j}}=\overrightarrow{b_{i} a_{i}}+\overrightarrow{a_{i} a_{j}}+\overrightarrow{a_{j} b_{j}}=\overrightarrow{a_{i} a_{j}} .
$$

LEMMA 3.9. - (i) For $i=0, \ldots, p, q_{n}\left(\overrightarrow{b_{i}}\right) \neq 0$ if

$$
\lambda^{2} \neq-\frac{q_{n}\left(\overrightarrow{a_{p}}\right) q_{n}\left(\overrightarrow{a_{i} a_{p}}\right)}{q_{n}\left(\overrightarrow{a_{i}}\right) q_{n}(\vec{w})} .
$$

(ii) For $0 \leqslant i<j \leqslant p, q_{n}\left(\overrightarrow{a_{i} b_{j}}\right) \neq 0$ if

$$
\lambda^{2} \neq-\frac{q_{n}\left(\overrightarrow{a_{p}}\right)\left(q_{n}\left(\overrightarrow{a_{i} a_{j}}\right)+q_{n}\left(\overrightarrow{a_{p}}\right)\right)}{q_{n}\left(\overrightarrow{a_{i} a_{j}}\right) q_{n}(\vec{w})} .
$$

Let us compute $q_{n}\left(\overrightarrow{b_{i}}\right)$. We have $\overrightarrow{b_{i}}=\overrightarrow{a_{p}}+\overrightarrow{a_{p} a_{i}}+\overrightarrow{a_{i} b_{i}}$. And since $\overrightarrow{a_{p} a_{i}}$ is orthogonal to $\overrightarrow{a_{p}}$ and $\overrightarrow{a_{i} b_{i}}$, we get

$4^{\text {e }}$ SÉRIE - TOME $40-2007-\mathrm{N}^{\circ} 3$ 


$$
\begin{aligned}
q_{n}\left(\overrightarrow{b_{i}}\right) & =q_{n}\left(\overrightarrow{a_{i}} \overrightarrow{a_{p}}\right)+q_{n}\left(\overrightarrow{a_{p}}\right)+q_{n}\left(\overrightarrow{a_{i} b_{i}}\right)+2\left\langle\overrightarrow{a_{p}}, \overrightarrow{a_{i} b_{i}}\right\rangle \\
& =q_{n}\left(\overrightarrow{a_{i} a_{p}}\right)+q_{n}\left(\overrightarrow{a_{p}}\right)-\frac{q_{n}\left(\overrightarrow{a_{p}}\right)^{2}}{q_{n}\left(\overrightarrow{w_{\lambda}}\right)} \\
& =q_{n}\left(\overrightarrow{a_{i}}\right)-\frac{q_{n}\left(\overrightarrow{a_{p}}\right)^{2}}{q_{n}\left(\overrightarrow{w_{\lambda}}\right)} .
\end{aligned}
$$

Finally, from the equality $q_{n}\left(\overrightarrow{a_{i}}\right)-q_{n}\left(\overrightarrow{a_{p}}\right)=q_{n}\left(\overrightarrow{a_{i} a_{p}}\right)$,

$$
q_{n}\left(\overrightarrow{b_{i}}\right)=\frac{\lambda^{2} q_{n}\left(\overrightarrow{a_{i}}\right) q_{n}(\vec{w})+q_{n}\left(\overrightarrow{a_{p}}\right) q_{n}\left(\overrightarrow{a_{i} a_{p}}\right)}{q_{n}\left(\overrightarrow{w_{\lambda}}\right)}
$$

This proves Part (i).

Since for $i<j, \overrightarrow{b_{j}}$ is orthogonal to $\overrightarrow{a_{i} b_{j}}$, we have $q_{n}\left(\overrightarrow{a_{i} b_{j}}\right)=q_{n}\left(\overrightarrow{a_{i}}\right)-q_{n}\left(\overrightarrow{b_{j}}\right)$. Using the previous calculation, we get

$$
q_{n}\left(\overrightarrow{a_{i} b_{j}}\right)=\frac{\lambda^{2} q_{n}\left(\overrightarrow{a_{i} a_{j}}\right) q_{n}(\vec{w})+q_{n}\left(\overrightarrow{a_{p}}\right)\left(q_{n}\left(\overrightarrow{a_{i} a_{j}}\right)+q_{n}\left(\overrightarrow{a_{p}}\right)\right)}{q_{n}\left(\overrightarrow{w_{\lambda}}\right)} .
$$

This gives the condition (ii).

LEMMA 3.10. - For $i \leqslant j$, we have

$$
\left\langle\overrightarrow{a_{i}}, \overrightarrow{a_{j}}\right\rangle=q_{n}\left(\overrightarrow{a_{j}}\right)
$$

and

$$
\left\langle\overrightarrow{a_{i}}, \overrightarrow{b_{j}}\right\rangle=\left\langle\overrightarrow{b_{i}}, \overrightarrow{b_{j}}\right\rangle=q_{n}\left(\overrightarrow{b_{j}}\right) .
$$

The first relation is already known. For the second, we use the equalities $\overrightarrow{a_{i}}=\overrightarrow{b_{j}}+\overrightarrow{b_{j} a_{i}}$ and $\overrightarrow{b_{i}}=\overrightarrow{b_{j}}+\overrightarrow{b_{j} b_{i}}$, as well as the fact that $\overrightarrow{b_{j}}$ is orthogonal to $\overrightarrow{b_{j} a_{i}}$ and $\overrightarrow{b_{j} b_{i}}$, for $i<j$.

LEMmA 3.11. - We assume that $\lambda$ satisfies the conditions of Lemma 3.9. For any $i \in$ $\{0,1, \ldots, p\}$, the simplex

$$
\left(\overrightarrow{a_{0}}, \ldots, \overrightarrow{a_{i}}, \overrightarrow{b_{i}}, \ldots, \overrightarrow{b_{p}}\right)
$$

is generic and geometric. In other words the vector $\overrightarrow{w_{\lambda}}$ is admissible.

This achieves the proof of Proposition 3.6.

For the proof of this lemma, it is enough to show that for

$$
0 \leqslant i_{0}<\cdots<i_{s}<i_{s+1}<\cdots<i_{t} \leqslant p,
$$

the Gram determinants

$$
\Delta_{s, t}=\operatorname{Gram}\left(\overrightarrow{a_{i_{0}}}, \ldots, \overrightarrow{a_{i_{s}}}, \overrightarrow{b_{i_{s}}}, \ldots, \overrightarrow{b_{i_{t}}}\right)
$$

and

$$
\Delta_{s, t}^{\prime}=\operatorname{Gram}\left(\overrightarrow{a_{i_{0}}}, \ldots, \overrightarrow{a_{i_{s}}}, \overrightarrow{b_{i_{s+1}}}, \ldots, \overrightarrow{b_{i_{t}}}\right)
$$

are not 0 . 
We need the following determinant

$$
D\left(\alpha_{1}, \ldots, \alpha_{l}\right):=\left|\begin{array}{ccccc}
\alpha_{1} & \alpha_{2} & \alpha_{3} & \ldots & \alpha_{l} \\
\alpha_{2} & \alpha_{2} & \alpha_{3} & \ldots & \alpha_{l} \\
\alpha_{3} & \alpha_{3} & \alpha_{3} & \ldots & \alpha_{l} \\
\cdot & \cdot & \cdot & \ldots & \cdot \\
\alpha_{l} & \alpha_{l} & \alpha_{l} & \ldots & \alpha_{l}
\end{array}\right|=\alpha_{l} \prod_{i=1}^{l-1}\left(\alpha_{i}-\alpha_{i+1}\right) .
$$

We get from Lemma 3.10, that

$$
\Delta_{s, t}=D\left(q_{n}\left(\overrightarrow{a_{i_{0}}}\right), q_{n}\left(\overrightarrow{a_{i_{1}}}\right), \ldots, q_{n}\left(\overrightarrow{a_{i_{s}}}\right), q_{n}\left(\overrightarrow{b_{i_{s}}}\right), \ldots, q_{n}\left(\overrightarrow{b_{i_{t}}}\right)\right)
$$

and

$$
\Delta_{s, t}^{\prime}=D\left(q_{n}\left(\overrightarrow{a_{i_{0}}}\right), q_{n}\left(\overrightarrow{a_{i_{1}}}\right), \ldots, q_{n}\left(\overrightarrow{a_{i_{s}}}\right), q_{n}\left(\overrightarrow{b_{i_{s+1}}}\right), \ldots, q_{n}\left(\overrightarrow{b_{i_{t}}}\right)\right) .
$$

And we finally deduce from the previous lemmas, that these determinants are not 0 , with the conditions imposed on $\lambda$. More precisely, we have proved that:

for $0 \leqslant i \leqslant p$,

$$
\begin{aligned}
& q_{n}\left(\overrightarrow{a_{i}}\right) \neq 0, \quad q_{n}\left(\overrightarrow{b_{i}}\right) \neq 0, \\
& q_{n}\left(\overrightarrow{a_{i}}\right)-q_{n}\left(\overrightarrow{b_{i}}\right)=q_{n}\left(\overrightarrow{a_{i} b_{i}}\right) \neq 0,
\end{aligned}
$$

and for $0 \leqslant i<j \leqslant p$,

$$
\begin{aligned}
& q_{n}\left(\overrightarrow{a_{i}}\right)-q_{n}\left(\overrightarrow{a_{j}}\right)=q_{n}\left(\overrightarrow{a_{i} a_{j}}\right) \neq 0, \\
& q_{n}\left(\overrightarrow{b_{i}}\right)-q_{n}\left(\overrightarrow{b_{j}}\right)=q_{n}\left(\overrightarrow{b_{i} b_{j}}\right) \neq 0, \\
& q_{n}\left(\overrightarrow{a_{i}}\right)-q_{n}\left(\overrightarrow{b_{j}}\right)=q_{n}\left(\overrightarrow{a_{i} b_{j}}\right) \neq 0 .
\end{aligned}
$$

We are now in a position to finish the proof of the extension property, for nice simplices. Let $\left(v_{0}, v_{1}, \ldots, v_{p}\right)$ be a nice simplex, with $p \leqslant n-2$. By Corollary 3.7 and Lemma 2.7, the set of points which are admissible for all the flags (see the basic example above)

$$
V_{0}^{\sigma} \subset V_{1}^{\sigma} \subset \cdots \subset V_{p}^{\sigma}
$$

is a non-empty Zariski open subset of $Q_{n}(\mathbf{k})$. But this open set $G$ coincides with the set of points $\omega \in Q_{n}(\mathbf{k})$, for which $\left(v_{0}, v_{1}, \ldots, v_{p}, \omega\right)$ is nice. One of the inclusions results from the definitions. To see the second consider $v_{p+1} \in G$; it is clear that the simplex $\left(v_{0}, v_{1}, \ldots, v_{p}, v_{p+1}\right)$ is strongly geometric. Now, fix a permutation $\sigma$ of $\{0,1, \ldots, p\}$. With the previous notations, the $p+1$ simplices $(i=0,1, \ldots, p)$

$$
\left(\mathbf{k} \overrightarrow{a_{0}}, \ldots, \mathbf{k} \overrightarrow{a_{i}}, \mathbf{k} \overrightarrow{b_{i}}, \ldots, \mathbf{k} \overrightarrow{b_{p}}\right)
$$

related to the flag: $V_{0}^{\sigma} \subset V_{1}^{\sigma} \subset \cdots \subset V_{p}^{\sigma}$, with vertex $v_{\sigma(0)}$ and admissible point $v_{p+1}$, are exactly the simplices $\left(L_{0}^{\mu}, L_{1}^{\mu}, \ldots, L_{p+1}^{\mu}\right)$, corresponding to $\left(v_{0}, v_{1}, \ldots, v_{p}, v_{p+1}\right)$, with $\mu$ one of the permutations

$$
\left(\begin{array}{ccccccccc}
0 & 1 & \ldots & i-1 & i & i+1 & \ldots & p & p+1 \\
\sigma(0) & \sigma(1) & \ldots & \sigma(i-1) & p+1 & \sigma(i) & \ldots & \sigma(p-1) & \sigma(p)
\end{array}\right)
$$

$4^{\text {e }}$ SÉRIE - TOME $40-2007-\mathrm{N}^{\circ} 3$ 
where $i=1,2, \ldots, p, p+1$. In this way, when $\sigma$ varies, we get all the simplices $\left(L_{0}^{\mu}, L_{1}^{\mu}, \ldots\right.$, $\left.L_{p+1}^{\mu}\right)$, such that $\mu(0) \in\{0,1, \ldots, p\}$. If $\mu(0)=p+1$ and $q=\mu(1)$, let $H_{q}$ be the linear subspace of codimension 1, orthogonal to $\overrightarrow{v_{p+1} v_{q}}$. By Proposition $2.2,\left(L_{0}^{\mu}, L_{1}^{\mu}, \ldots, L_{p+1}^{\mu}\right)$ is symmetric to one of the previous simplices, through the orthogonal symmetry $s_{H_{q}}$. Therefore this simplex is generic and geometric. But so far, we have exhausted all the simplices $\left(L_{0}^{\mu}, L_{1}^{\mu}, \ldots, L_{p+1}^{\mu}\right)$, for the simplex $\left(v_{0}, v_{1}, \ldots, v_{p}, v_{p+1}\right)$. This proves that $\left(v_{0}, v_{1}, \ldots, v_{p}, v_{p+1}\right)$ is nice, and achieves the proof of the extension property.

Remark 3.12. - The proofs in this section use only the fact that $\mathrm{k}$ is infinite. As a result, Proposition 2.6 and Theorem 2.5 are true for any infinite field $\mathrm{k}$ of characteristic not 2 .

\section{Steinberg modules}

\subsection{Tits buildings}

In this subsection we could work equally well with any non-degenerate quadratic space as in [9], and the hypothesis $\mathbf{k}$ Pythagorean is not necessary.

DEFINITION 4.1. - We define the "Tits simplicial set" $\mathcal{T}(n)$ of $\left(\mathbf{k}^{n}, q_{n}\right)$, as the nerve of the category of non-zero strict non-degenerate linear subspaces of $\mathbf{k}^{n}$, with morphisms given by inclusions.

In particular, a $p$-simplex is a flag $U_{0} \subset U_{1} \subset \cdots \subset U_{p}$ of non-degenerate linear subspaces of $\mathbf{k}^{n}$, with the conditions $U_{0} \neq 0$ and $U_{p} \neq \mathbf{k}^{n}$. The faces and degeneracies are given by

$$
\begin{gathered}
U_{0} \subset U_{1} \subset \cdots \subset U_{p} \mapsto U_{0} \subset \cdots \subset \hat{U}_{i} \subset \cdots \subset U_{p}, \\
U_{0} \subset U_{1} \subset \cdots \subset U_{p} \mapsto U_{0} \subset \cdots \subset U_{i} \subset U_{i} \subset \cdots \subset U_{p} .
\end{gathered}
$$

We denote by $C_{*}(\mathcal{T}(n), \partial)$ the singular complex of $\mathcal{T}(n)$.

PROPOSITION 4.2. - For $n \geqslant 2$, the homology of the simplicial set $\mathcal{T}(n)$ satisfies

$$
\tilde{H}_{i}(\mathcal{T}(n), \mathbb{Z})=0, \quad \text { for } i \neq n-2 .
$$

Proof. - We proceed along the lines of [15] Theorem 3.5. Since a non-degenerate simplex is of the form $U_{0} \varsubsetneqq U_{1} \varsubsetneqq \cdots \varsubsetneqq U_{p}$, there is no non-degenerate $p$-simplex for $p>n-2$, and then

$$
\tilde{H}_{i}(\mathcal{T}(n), \mathbb{Z})=0, \quad \text { for } i>n-2 .
$$

Let $B_{p, q}$ be the free $\mathbb{Z}$-module generated by the couples $\left(\left(L_{0}, \ldots, L_{p}\right), U_{0} \subset U_{1} \subset \cdots \subset U_{q}\right)$, where $U_{0} \subset U_{1} \subset \cdots \subset U_{p}$ is a non-degenerate flag, and $\left(L_{0}, \ldots, L_{p}\right)$ is a geometric simplex of lines in $U_{0}$. The two spectral sequences of the bicomplex $\left(B_{*, *, d, \partial}\right)$ collapse. Actually, we have exact augmented complexes

$$
\begin{aligned}
& \cdots \stackrel{\partial}{\rightarrow} B_{*, q}(n) \stackrel{\partial}{\rightarrow} \cdots \stackrel{\partial}{\rightarrow} B_{*, 0}(n) \stackrel{\eta}{\rightarrow} B_{*}^{\prime}(n) \rightarrow 0, \\
& \ldots \stackrel{d}{\rightarrow} B_{p, *}(n) \stackrel{d}{\rightarrow} \cdots \stackrel{d}{\rightarrow} B_{0, *}(n) \stackrel{\epsilon}{\rightarrow} C_{*}(\mathcal{T}(n)) \rightarrow 0 .
\end{aligned}
$$

The exactness of (3) stems from Theorem 2.5, and for the complex (2) there is a contracting homotopy $h$, given by

$$
h\left(\left(L_{0}, \ldots, L_{p}\right), U_{0} \subset U_{1} \subset \cdots \subset U_{q}\right)=\left(\left(L_{0}, \ldots, L_{p}\right), W \subset U_{0} \subset U_{1} \subset \cdots \subset U_{q}\right),
$$


where $W=\sum_{i} L_{i}$. Note that the map $\epsilon$ is surjective.

As a consequence, for $i \geqslant 0$, we get isomorphisms

$$
\tilde{H}_{i}(\mathcal{T}(n), \mathbb{Z}) \cong \tilde{H}_{i}\left(B_{*}^{\prime}(n)\right) \cong H_{i+1}\left(\hat{B}_{*}(n)\right) .
$$

But these groups are respectively reduced to 0 , for $i>n-2$ by (1) above, and for $i<n-2$, by Corollary 2.8 .

It is amusing to check directly that $\tilde{H}_{0}(\mathcal{T}(n), \mathbb{Z})=0$, for $n>2$.

\subsection{Coinvariants of Steinberg modules}

Definition 4.3. - We define the Steinberg $O(n)$-module $S t(n)$, by

$$
S t(0)=S t(1)=\mathbb{Z},
$$

and

$$
S t(n)=\tilde{H}_{n-2}(\mathcal{T}(n), \mathbb{Z}), \quad \text { for } n \geqslant 2 .
$$

We proceed now to prove Theorem 1.2, asserting that

$$
H_{0}(O(n), S t(n))=0, \quad \text { for } n \geqslant 2,
$$

when $\mathbf{k}$ is Pythagorean.

It is interesting to give a simple direct proof, in the particular case $n=2$. We use the fact that for any anisotropic line $U, U \cap Q_{2}(\mathbf{k})$ is not empty. One observes firstly that $S t(2)$ is generated by the differences $U_{1}-U_{2}$, where $U_{1}$ and $U_{2}$ are anisotropic lines. Since $\mathbf{k}$ is infinite, one can find an anisotropic line $U_{3}$, such that for a choice of $u_{i} \in U_{i} \cap Q_{2}(\mathbf{k}), u_{1}+u_{3}$ and $u_{2}+u_{3}$ are anisotropic. The orthogonal symmetry, around the anisotropic line $V_{1}=\mathbf{k}\left(u_{1}+u_{3}\right)$, exchanges $U_{1}$ and $U_{3}$. As a result, in the group of coinvariants, one gets $U_{1}-V_{1}=U_{3}-V_{1}$ and then $U_{1}-U_{3}=0$. Proceeding in the same way, with $V_{2}=\mathbf{k}\left(u_{2}+u_{3}\right)$, one gets $U_{3}-U_{2}=0$. This proves that $U_{1}-U_{2}=0$, in $H_{0}(O(2), S t(2))$.

We can be more precise.

Proposition 4.4. - We have $H_{0}(O(2), S t(2))=0$, iff $\mathbf{k}$ is Pythagorean.

Let $\mathfrak{G}$ be the subgroup of $\mathbf{k}^{\times} /\left(\mathbf{k}^{\times}\right)^{2}$ generated by the elements which are the sum of two squares. This group is reduced to 1 , iff $\mathbf{k}$ is Pythagorean. Now one checks easily that there is a surjection

$$
H_{0}(O(2), S t(2)) \rightarrow \mathfrak{G}
$$

induced by

$$
U_{1}-U_{2} \mapsto\left(x_{1}^{2}+y_{1}^{2}\right) /\left(x_{2}^{2}+y_{2}^{2}\right),
$$

where $\left(x_{1}, y_{1}\right)$ and $\left(x_{2}, y_{2}\right)$ are non-zero vectors of $U_{1}$ and $U_{2}$.

The proof of Theorem 1.2 in full generality combines the projective and excellent complexes. We begin with a byproduct of the proof of Proposition 4.2, where the coinvariants of an $O(n)$-module $M$ are denoted by $M_{O(n)}$.

PROPOSITION 4.5. - For $n \geqslant 2$, there is an exact sequence of groups of coinvariants

$$
\hat{C}_{n}(n)_{O(n)} \stackrel{d}{\rightarrow} \hat{C}_{n-1}(n)_{O(n)} \rightarrow S t(n)_{O(n)} \rightarrow 0
$$

$4^{\mathrm{e}}$ SÉRIE - TOME $40-2007-\mathrm{N}^{\circ} 3$ 
and a commutative diagram with exact rows

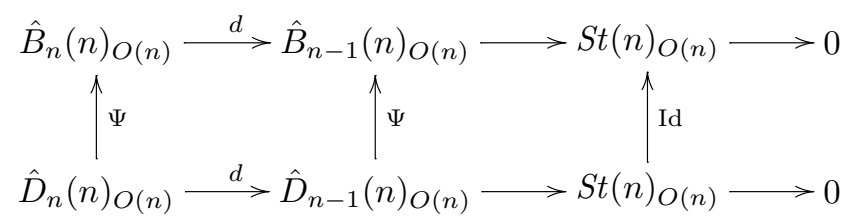

For $\mathbf{k}$ Pythagorean, the proof of Proposition 4.2 works equally well for the three complexes, giving exact sequences

$$
\begin{aligned}
& \hat{C}_{n}(n) \stackrel{d}{\rightarrow} \hat{C}_{n-1}(n) \rightarrow S t(n) \rightarrow 0, \\
& \hat{B}_{n}(n) \stackrel{d}{\rightarrow} \hat{B}_{n-1}(n) \rightarrow S t(n) \rightarrow 0, \\
& \hat{D}_{n}(n) \stackrel{d}{\rightarrow} \hat{D}_{n-1}(n) \rightarrow S t(n) \rightarrow 0 .
\end{aligned}
$$

To finish, we apply the right exact functor $(.)_{O(n)}$.

Remark 4.6. - This is the point where the hypothesis: $\mathbf{k}$ is Pythagorean, is essential. In the versions of the complex (3) for $C_{*}$ and $D_{*}$, the map $\epsilon$ is not surjective in general. For the surjectivity, any non-degenerate linear subspace of dimension 1 of $\mathbf{k}^{n}$, with $n \geqslant 2$, has to be Euclidean. But already for $n=2$, this implies that $\mathbf{k}$ is Pythagorean.

Theorem 1.2 results from the lemma

LEMMA 4.7. - The morphism induced by $\Psi$

$$
\hat{D}_{n-1}(n)_{O(n)} / d \hat{D}_{n}(n)_{O(n)} \longrightarrow \hat{B}_{n-1}(n)_{O(n)} / d \hat{B}_{n}(n)_{O(n)},
$$

is equal to 0 .

Of course this assertion is equivalent to the equality $\operatorname{Id}_{H_{0}(O(n), S t(n))}=0$, which is the theorem.

Now the lemma is an avatar of the "circumcenter argument" of Sah [33]. We start from a nice simplex of rank $n, \omega=\left(v_{0}, v_{1}, \ldots, v_{n-1}\right)$. With the notations of Section 2 , we have

$$
\Psi\left(\left(v_{0}, v_{1}, \ldots, v_{n-1}\right)\right)=\left(L_{\{0\}}, L_{\{1\}}, \ldots, L_{\{n-1\}}\right) .
$$

We now stay in the complex $B_{*}(n)$. Modulo a boundary, we can write

$$
\Psi(\omega)=\sum_{i=0}^{n-1}(-1)^{i+n-1}\left(L_{\{0\}}, \ldots, \widehat{L_{\{i\}}}, \ldots, L_{\{n-1\}}, L_{\{0,1, \ldots, n-1\}}\right) .
$$

An induction shows that for $j \geqslant 1$, up to boundaries and modulo simplices in $B_{*}^{\prime}(n), \Psi(\omega)$ can be written as a sum (with coefficients \pm 1 ) of simplices in $B_{n-1}(n)$, of the type

$$
\left(L_{\left\{i_{0}\right\}}, L_{\left\{i_{1}\right\}}, \ldots, L_{\left\{i_{j}\right\}}, L_{I_{j+1}}, \ldots, L_{I_{n-1}}\right) .
$$

Here

- $I_{i}$ is a subset of $\{0,1, \ldots, n-1\}$ with $i+1$ elements,

- $\left\{i_{0}<i_{1}<\cdots<i_{j}\right\} \subset I_{j+1}$, 
$-I_{j+1} \subset I_{j+2} \subset \cdots \subset I_{n-1}$

(incidentally, for $j=0$, these data are in bijection with the permutations of $\{0,1, \ldots, n-1\}$ ).

For $j=1$, we arrive at simplices of the type

$$
\left(L_{\left\{i_{0}\right\}}, L_{\left\{i_{1}\right\}}, L_{I_{2}}, L_{I_{3}}, \ldots, L_{I_{n-1}}\right) .
$$

Let us detail the end of the proof. We want to show that such a simplex is equal to 0 , in the group of coinvariants $\hat{B}_{n-1}(n)_{O(n)}$. If we put $I_{1}=\left\{i_{0}, i_{1}\right\}$, we have in the complex $\hat{B}_{*}(n)$

$$
\begin{aligned}
& d\left(L_{\left\{i_{0}\right\}}, L_{\left\{i_{1}\right\}}, L_{I_{1}}, L_{I_{2}}, \ldots, L_{I_{n-1}}\right)=\left(L_{\left\{i_{1}\right\}}, L_{I_{1}}, L_{I_{2}}, L_{I_{3}}, \ldots, L_{I_{n-1}}\right) \\
& \quad-\left(L_{\left\{i_{0}\right\}}, L_{I_{1}}, L_{I_{2}}, L_{I_{3}}, \ldots, L_{I_{n-1}}\right)+\left(L_{\left\{i_{0}\right\}}, L_{\left\{i_{1}\right\}}, L_{I_{2}}, L_{I_{3}}, \ldots, L_{I_{n-1}}\right) .
\end{aligned}
$$

Thus modulo boundaries

$$
\begin{aligned}
& \left(L_{\left\{i_{0}\right\}}, L_{\left\{i_{1}\right\}}, L_{I_{2}}, L_{I_{3}}, \ldots, L_{I_{n-1}}\right) \\
& \quad=\left(L_{\left\{i_{0}\right\}}, L_{I_{1}}, L_{I_{2}}, L_{I_{3}}, \ldots, L_{I_{n-1}}\right)-\left(L_{\left\{i_{1}\right\}}, L_{I_{1}}, L_{I_{2}}, L_{I_{3}}, \ldots, L_{I_{n-1}}\right) .
\end{aligned}
$$

If we consider now the orthogonal symmetry $s_{H}$, corresponding to the linear hyperplane $H$ orthogonal to $\overrightarrow{v_{i_{0}} v_{i_{1}}}$, we get

$$
s_{H}\left(\left(L_{\left\{i_{0}\right\}}, L_{I_{1}}, L_{I_{2}}, L_{I_{3}}, \ldots, L_{I_{n-1}}\right)\right)=\left(L_{\left\{i_{1}\right\}}, L_{I_{1}}, L_{I_{2}}, L_{I_{3}}, \ldots, L_{I_{n-1}}\right),
$$

because $L_{I_{i}} \subset H$, for $i \geqslant 1$. This shows that $\left(L_{\left\{i_{0}\right\}}, L_{\left\{i_{1}\right\}}, L_{I_{2}}, L_{I_{3}}, \ldots, L_{I_{n-1}}\right)$ is 0 , in the group of coinvariants $\hat{B}_{n-1}(n)_{O(n)}$.

The following proposition is used in the next section.

PROPOSITION 4.8. - The homology of the complex of coinvariants stemming from the quotient complex $\hat{C}_{*}(n)$ satisfies

$$
H_{n-1}\left(\hat{C}_{*}(n)_{O(n)}\right)=0, \text { for } n \geqslant 2 .
$$

In fact this group is equal to $H_{0}(O(n), S t(n))$, by Proposition 4.5.

\section{Homology stability for $O(n)$}

The proof of Theorem 1.1 follows the arguments of Sah in [33], Section 1.

The filtration of the complex $C_{*}(n)$, by the rank of the simplices, induces a filtration $\mathcal{F}_{j}^{r}(n)$ of the complex $H_{j}\left(O(n), C_{*}(n)\right)$. In particular $\mathcal{F}_{0}^{n}(n)=C_{*}(n)_{O(n)}$, and $\mathcal{F}_{0}^{n-1}(n)$ is the image of the complex $C_{*}^{\prime}(n)_{O(n)}$ in $C_{*}(n)_{O(n)}$. The following lemma uses the "Orthogonal Join Construction" of Sah [33] ${ }^{5}$.

LEMma 5.1. - (i) The complex $\mathcal{F}_{0}^{r}(n)$ is $(r-2)$-acyclic with augmentation $\mathbb{Z}$, for $1 \leqslant r \leqslant n$. (ii) Furthermore, for $r \geqslant 2$,

$$
H_{r-1}\left(\mathcal{F}_{0}^{r}(n) / \mathcal{F}_{0}^{r-1}(n)\right)=0
$$

We recall briefly the arguments. Let $e_{1}, \ldots, e_{n}$ be the canonical basis of $\mathbf{k}^{n}$. For $m \leqslant n$, we identify $\mathbf{k}^{m}$ with the linear space generated by the $e_{1}, \ldots, e_{m}$. For $p \leqslant r-2$, an element

\footnotetext{
${ }^{5}$ Unfortunately, we were unable to find any good complex, generated by simplices with circumcenters, for which the orthogonal join construction works.

$4^{\text {e }}$ SÉRIE - TOME $40-2007-\mathrm{N}^{\circ} 3$
} 
of $H_{p}\left(\mathcal{F}_{0}^{r}(n)\right)$ is represented by a chain $c \in C_{p}(r-1)$. An application of the Witt extension theorem shows that we have in $\mathcal{F}_{0}^{r}(n): d\left(e_{r} \star c\right)=c$, where $e_{r} \star c$ is the join of $e_{r}$ with $c$, and this join is geometric. This proves Part (i) of the lemma.

The second part is a corollary of Proposition 4.8. One checks easily that the natural morphism of complexes

$$
\hat{C}_{*}(r)_{O(r)} \rightarrow \mathcal{F}_{0}^{r}(n) / \mathcal{F}_{0}^{r-1}(n)
$$

induces a surjection

$$
H_{r-1}\left(\hat{C}_{*}(r)_{O(r)}\right) \rightarrow H_{r-1}\left(\mathcal{F}_{0}^{r}(n) / \mathcal{F}_{0}^{r-1}(n)\right)
$$

For this, one remarks that these complexes are 0 , in degree $\leqslant r-2$, and in degree $r-1$ the chains are generated by the simplices of rank $r$.

PROPOSITION 5.2. - The complex $\mathcal{F}_{0}^{r}(n)$ is $(r-1)$-acyclic, for $1 \leqslant r \leqslant n$.

One applies the lemma to the long homology sequence of the short exact sequence of complexes

$$
0 \rightarrow \mathcal{F}_{0}^{r-1}(n) \rightarrow \mathcal{F}_{0}^{r}(n) \rightarrow \mathcal{F}_{0}^{r}(n) / \mathcal{F}_{0}^{r-1}(n) \rightarrow 0 .
$$

One settles first the case $r=n$, using the fact that the maps for $i>0$

$$
H_{i}\left(\mathcal{F}_{0}^{n-1}(n)\right) \rightarrow H_{i}\left(\mathcal{F}_{0}^{n}(n)\right)
$$

are zero, by the orthogonal join construction.

Now the proof of Theorem 1.1 is connected to the proof of the following proposition.

PROPOSITION 5.3. - From the acyclicity of the complex of $O(n)$-modules $C_{*}(n)$, the hyperhomology $\mathbb{H}_{i}\left(O(n), C_{*}(n)\right)$ is equal to $H_{i}(O(n), \mathbb{Z})$. The hyperhomology spectral sequence

$$
E_{i, j}^{1}=H_{j}\left(O(n), C_{i}(n)\right) \Longrightarrow H_{i+j}(O(n), \mathbb{Z})
$$

satisfies

(i) $E_{0, j}^{1}=H_{j}(O(n-1), \mathbb{Z})$, for $j \geqslant 0$, and the edge morphism $E_{0, j}^{1} \rightarrow H_{j}(O(n), \mathbb{Z})$, is canonically identified to the stabilization map

$$
H_{j}(O(n-1), \mathbb{Z}) \rightarrow H_{j}(O(n), \mathbb{Z}) .
$$

(ii) $E_{i, j}^{2}=0$, for $1 \leqslant i \leqslant n-j-1$.

From (ii), $E_{0, j}^{2} \rightarrow E_{0, j}^{\infty}$ is bijective for $j<n-1$, and surjective for $j=n-1$. Also, the edge morphism $E_{0, j}^{\infty} \rightarrow H_{j}(O(n), \mathbb{Z})$ is bijective for $j \leqslant n-1$. Therefore, Theorem 1.1 is a corollary of the proposition, for the case $n+1$, instead of $n$.

Part (i) of the proposition is independent of the theorem. The free $\mathbb{Z}$-module $C_{0}(n)$ is a permutation module under the action of $O(n)$, with basis composed of one orbit. By Schapiro's lemma ([5] Chap. 3), $E_{0, j}^{1}=H_{j}(O(n-1), \mathbb{Z})$, where $O(n-1)$ is taken to be the stabilizer of $e_{n}$. Now the differential $d^{1}: E_{1, j}^{1} \rightarrow E_{0, j}^{1}$ is the zero map. Actually, by Schapiro's lemma, an element in $E_{1, j}^{1}$ can be represented, up to signs, by a sum of terms of the type $c \otimes\left(v_{0}, v_{1}\right)$, where $\left(v_{0}, v_{1}\right)$ is a geometric simplex, and $c$ is a $j$-cycle in the bar complex of the stabilizer $G_{\left(v_{0}, v_{1}\right)}$ of $\left(v_{0}, v_{1}\right)$. We have, up to sign, $d^{1}\left(c \otimes\left(v_{0}, v_{1}\right)\right)=c \otimes v_{1}-c \otimes v_{0}$. One can find an element $f \in O(n)$, such 
that $f\left(v_{0}\right)=v_{1}$, with $c$ fixed by conjugation by $f$. This shows that $d^{1}\left(c \otimes\left(v_{0}, v_{1}\right)\right)=0$, by a well known fact in homology of groups.

Proof of (ii). One proceeds by induction, starting from the case $n=1$ of Proposition 5.3. Assume the proposition proved, for $l<n$. Then the theorem is also true for $l<n-1$, by the above remark. We want to prove that the complex $\left(E_{*, j}^{1}, d^{1}\right)$ is acyclic in degrees: $1 \leqslant i \leqslant n-j-1$. This is the complex

$$
\cdots \stackrel{d}{\rightarrow} H_{j}\left(O(n), C_{i}(n)\right) \stackrel{d}{\rightarrow} \cdots \stackrel{d}{\rightarrow} H_{j}\left(O(n), C_{0}(n)\right) .
$$

We already know that its homology, in degree 0 , is $H_{j}(O(n-1), \mathbb{Z})$. To prove the acyclicity, it is enough to consider the subcomplex $\mathcal{F}_{j}^{n-j}(n)$ in the interval of interest, that is for $0 \leqslant i \leqslant n-j$, because the two complexes coincide in the interval $0 \leqslant i \leqslant n-j-1$. Also we have to consider the $j$ such that: $j<n-1$. To finish the proof, we use the following lemma.

LEMMA 5.4. - There exists an exact sequence

$$
0 \rightarrow A(j) \otimes \mathcal{F}_{0}^{n-j-1}(n) \rightarrow H_{j}(O(j), \mathbb{Z}) \otimes \mathcal{F}_{0}^{n-j}(n) \rightarrow \mathcal{F}_{j}^{n-j}(n) \rightarrow 0,
$$

where $A(j)$ is the kernel of the map: $H_{j}(O(j), \mathbb{Z}) \rightarrow H_{j}(O(j+1), \mathbb{Z})$, which is surjective for $j \leqslant n-2$, by induction.

Proof of the lemma. - By Schapiro's lemma, $H_{j}\left(O(n), C_{i}(n)\right)$ can be written as a direct sum of groups of the type $H_{j}(O(r), \mathbb{Z}) \otimes C_{i}(n)_{n-r}$, where $C_{i}(n)_{n-r}$ means the coinvariants of the submodule of $C_{i}(n)$, generated by the simplices of rank: $n-r$. In our subcomplex $\mathcal{F}_{j}^{n-j}(n)$, we have $r \geqslant j$. By induction $H_{j}(O(r), \mathbb{Z})=H_{j}(O(j+1), \mathbb{Z})$, for $r>j$. The case where $r=j$ appears for the simplices of rank $j$, for which we have the exact sequence,

$$
0 \rightarrow A(j) \rightarrow H_{j}(O(j), \mathbb{Z}) \rightarrow H_{j}(O(j+1), \mathbb{Z}) \rightarrow 0 .
$$

This gives easily the searched exact sequence.

Finally, to prove that $\mathcal{F}_{j}^{n-j}(n)$ is $(n-j-1)$-acyclic, one applies Proposition 5.2 to the long homology sequence of the short exact sequence of the lemma, using the fact that $\mathcal{F}_{0}^{r}(n)$ is a free $\mathbb{Z}$-module.

\section{Homology stability for $S O(n)$}

\subsection{A spectral sequence}

The purpose of this section is the proof of Theorem 1.4(ii). Here is a basic elementary result.

Proposition 6.1. - (i) In degree 0

$$
\begin{aligned}
& H_{0}\left(O(2), \mathbb{Z}^{t}\right)=\mathbb{Z} / 2 \mathbb{Z}, \\
& H_{0}\left(O(2), \mathbb{Z}[1 / 2]^{t}\right)=0 .
\end{aligned}
$$

(ii) In degree 1

$$
\begin{aligned}
& H_{1}\left(O(2), \mathbb{Z}^{t}\right) \cong H_{1}(S O(2), \mathbb{Z}) \cong S O(2), \\
& H_{1}\left(O(2), \mathbb{Z}[1 / 2]^{t}\right) \cong H_{1}(S O(2), \mathbb{Z}[1 / 2]) \cong S O(2) \otimes \mathbb{Z}[1 / 2] .
\end{aligned}
$$

$4^{\mathrm{e}}$ SÉRIE - TOME $40-2007-\mathrm{N}^{\circ} 3$ 
One can use the Hochschild-Serre spectral sequence of the semi-direct extension

$$
0 \rightarrow S O(2) \rightarrow O(2) \rightarrow\{ \pm 1\} \rightarrow 0
$$

with coefficients in $\mathbb{Z}^{t}$, and remark that the natural action of $\{ \pm 1\}$ on $S O(2)$ arising from this extension, is given by $x \mapsto x^{-1}$. Note also that $\mathbb{Z}[1 / 2]$ is $\mathbb{Z}$-flat.

We give now another description of the Steinberg module.

DEFINITION 6.2. - For the quadratic space $\left(\mathbf{k}^{n}, q_{n}\right)$, we define the quasi-simplicial ${ }^{6}$, $\mathbb{Z}$-module: $[p] \rightarrow \mathcal{L}_{p}(n)$, as follows:

(i) $\mathcal{L}_{0}(0)=\mathbb{Z}$.

(ii) For $n \neq 0, \mathcal{L}_{p}(n)=0$ if $p=0$ or $p>n$.

(iii) Otherwise, $\mathcal{L}_{p}(n)$ is the free $\mathbb{Z}$-module generated by the symbols $\left(E_{1}, \ldots, E_{p}\right)$, where $\mathbf{k}^{n}=E_{1} \oplus \cdots \oplus E_{p}$ is an orthogonal direct sum decomposition by non-zero and nondegenerate subspaces.

(iv) The faces are given by the maps $\delta_{0}=0, \delta_{p}=0$ and $\delta_{i}:\left(E_{1}, \ldots, E_{p}\right) \mapsto\left(E_{1}, \ldots, E_{i} \oplus\right.$ $\left.E_{i+1}, \ldots, E_{p}\right)$, for $i=1, \ldots, p-1$.

The associated chain complex is written $\left(\mathcal{L}_{*}(n), \delta\right)$.

LEMmA 6.3. - For $n \geqslant 0, H_{n}\left(\mathcal{L}_{*}(n)\right)=S t(n)$, and $H_{i}\left(\mathcal{L}_{*}(n)\right)=0$ if $i \neq n$.

Actually, for $n \geqslant 2$, the complex $\left(\mathcal{L}_{*}(n), \delta\right)$ is in bijection with the double suspension of $C_{*}(\mathcal{T}(n), \partial)$, augmented to $\mathbb{Z}$. To see this fact, one associates to the $p$-tuple $\left(E_{1}, \ldots, E_{p}\right)$, the flag $U_{0} \subset U_{1} \subset \cdots \subset U_{p-2}$, with $U_{i}=E_{1} \oplus \cdots \oplus E_{i+1}$.

For what follows, the complex $\left(\mathcal{L}_{*}(n), \delta\right)$ is more convenient than $C_{*}(\mathcal{T}(n))$.

PROPOSITION 6.4. - For $n=2 m$, there is a spectral sequence

$$
E_{p, q}^{1}=\sum_{n_{1}+\cdots+n_{p}=n} H_{q}\left(O\left(n_{1}\right) \times \cdots \times O\left(n_{p}\right), \mathbb{Z}[1 / 2]^{t}\right) \Rightarrow H_{p+q-n}\left(O(n), \mathcal{S} t(n) \otimes \mathbb{Z}[1 / 2]^{t}\right),
$$

where the integers $n_{i}$ are even and $\neq 0$.

Proof. - We start from the hyperhomology groups

$$
\mathbb{H}_{*}\left(O(n), \mathcal{L}_{*}(n) \otimes \mathbb{Z}[1 / 2]^{t}\right) .
$$

These are the homology groups of the double complex

$$
\left(C_{s}\left(O(n), \mathcal{L}_{r}(n) \otimes \mathbb{Z}[1 / 2]^{t}\right), d, \delta\right),
$$

where $C_{*}$ means the non-homogeneous chain complex, in homology of groups, $d$ being here the corresponding differential. The differential $\delta$ is the one coming from the complex $\mathcal{L}_{*}(n)$.

The spectral sequence of this bicomplex, corresponding to the filtration by degree $s$, collapses from $E^{1}$. In fact, since $E_{p, q}^{1}=C_{p}\left(O(n), H_{q}\left(\mathcal{L}_{*}(n) \otimes \mathbb{Z}[1 / 2]^{t}\right)\right)$, we get $E_{p, q}^{1}=0$ for $q \neq n$, and $E_{p, n}^{1}=C_{p}\left(O(n), \mathcal{S} t(n) \otimes \mathbb{Z}[1 / 2]^{t}\right)$. Therefore we have $E_{p, n}^{2}=E_{p, n}^{\infty}=H_{p}(O(n), \mathcal{S} t(n) \otimes$ $\left.\mathbb{Z}[1 / 2]^{t}\right)$, so that

$$
\mathbb{H}_{i}\left(O(n), \mathcal{L}_{*}(n) \otimes \mathbb{Z}[1 / 2]^{t}\right) \cong H_{i-n}\left(O(n), \mathcal{S} t(n) \otimes \mathbb{Z}[1 / 2]^{t}\right) .
$$

\footnotetext{
${ }^{6}$ That is without degeneracies.
} 
In the other spectral sequence of the bicomplex (4), the $E^{1}$ term is

$$
E_{p, q}^{1}=H_{q}\left(O(n), \mathcal{L}_{p}(n) \otimes \mathbb{Z}[1 / 2]^{t}\right) .
$$

The basic fact is that the $O(n)$-module $\mathcal{L}_{p}(n) \otimes \mathbb{Z}[1 / 2]^{t}$ splits as a direct sum of induced modules, along the orbits, for the action of $O(n)$ on the set of orthogonal direct-sum decompositions of $\mathbf{k}^{n}$ into $p$ non-zero and non-degenerate linear subspaces. These orbits are parametrized by the $p$-tuples $\left(n_{1}, \ldots, n_{p}\right)$ of non-zero integers with $n_{1}+\cdots+n_{p}=n$, and the stabilizer of the associated canonical decomposition is $O\left(n_{1}\right) \times \cdots \times O\left(n_{p}\right)$. An application of Shapiro's lemma gives an isomorphism

$$
E_{p, q}^{1}=\sum_{n_{1}+\cdots+n_{p}=n} H_{q}\left(O\left(n_{1}\right) \times \cdots \times O\left(n_{p}\right), \mathbb{Z}[1 / 2]^{t}\right),
$$

the restriction on the parity of the integers $n_{i}$ comes from the Künneth exact sequence and the fact that $H_{i}\left(O(n), \mathbb{Z}[1 / 2]^{t}\right)=0$, for $n$ odd.

We can now prove Theorem 1.4(ii). We proceed by induction on the dimension $2 m$. By Proposition 6.1

$$
H_{0}\left(O(2), \mathbb{Z}[1 / 2]^{t}\right)=0
$$

Assume that we have proved that

$$
H_{q}\left(O\left(2 l_{1}\right) \times \cdots \times O\left(2 l_{p}\right), \mathbb{Z}[1 / 2]^{t}\right)=0
$$

for $\left(l_{1}, \ldots, l_{p}\right)$ and $q$, such that $l_{i} \neq 0$ and $0 \leqslant q<l_{1}+\cdots+l_{p} \leqslant m-1$.

Let $m_{1}+\cdots+m_{p}=m$, and $n_{i}=2 m_{i}$ the Künneth exact sequence, with $q<m$ and $p>1$,

$$
\begin{aligned}
0 & \rightarrow \sum_{\substack{i+j=q \\
j<m_{2}+\cdots+m_{p}}} H_{i}\left(O\left(n_{1}\right), \mathbb{Z}[1 / 2]^{t}\right) \otimes H_{j}\left(O\left(n_{2}\right) \times \cdots \times O\left(n_{p}\right), \mathbb{Z}[1 / 2]^{t}\right) \\
\rightarrow & H_{q}\left(O\left(n_{1}\right) \times \cdots \times O\left(n_{p}\right), \mathbb{Z}[1 / 2]^{t}\right) \\
& \rightarrow \sum_{\substack{r+s=q-1 \\
s<m_{2}+\cdots+m_{p}}} \operatorname{Tor}\left(H_{r}\left(O\left(n_{1}\right), \mathbb{Z}[1 / 2]^{t}\right), H_{s}\left(O\left(n_{2}\right) \times \cdots \times O\left(n_{p}\right), \mathbb{Z}[1 / 2]^{t}\right)\right),
\end{aligned}
$$

implies that

$$
H_{q}\left(O\left(n_{1}\right) \times \cdots \times O\left(n_{p}\right), \mathbb{Z}[1 / 2]^{t}\right)=0, \quad \text { for } q<m .
$$

In the previous spectral sequence, we have

$$
E_{p, q}^{1}=0, \quad \text { for } q<m, \text { and } p>1 .
$$

Therefore

$$
H_{q}\left(O(n), \mathbb{Z}[1 / 2]^{t}\right)=E_{1, q}^{1}=E_{1, q}^{\infty}=0 .
$$

This achieves the proof of Theorem 1.4(ii).

As observed in the introduction, Theorem 1.3 is a corollary of Theorems 1.1 and 1.4.

$4^{\text {e }}$ SÉRIE - TOME $40-2007-\mathrm{N}^{\circ} 3$ 


\subsection{A quadratic algebra}

This section is a preliminary to the proof of Theorem 1.5. It generalizes slightly some results in $[8,9]$. Let

$$
\mathbf{B}=\sum_{m \geqslant 0} H_{m}\left(O(2 m), \mathbb{Z}[1 / 2]^{t}\right) .
$$

The $\mathbb{Z}[1 / 2]$-module $\mathbf{B}$ is provided with the structure of a graded algebra by the compositions

$$
\begin{aligned}
& H_{m_{1}}\left(O\left(2 m_{1}\right), \mathbb{Z}[1 / 2]^{t}\right) \otimes H_{m_{2}}\left(O\left(2 m_{2}\right), \mathbb{Z}[1 / 2]^{t}\right) \\
& \stackrel{\phi}{\rightarrow} H_{m_{1}+m_{2}}\left(O\left(2 m_{1}\right) \times O\left(2 m_{2}\right), \mathbb{Z}[1 / 2]^{t} \otimes \mathbb{Z}[1 / 2]^{t}\right) \\
& \stackrel{\psi}{\rightarrow} H_{m_{1}+m_{2}}\left(O\left(2\left(m_{1}+m_{2}\right)\right), \mathbb{Z}[1 / 2]^{t}\right),
\end{aligned}
$$

where the first map is the Eilenberg-Zilber cross-product [5], and the second one comes from the diagonal embedding $O\left(2 m_{1}\right) \times O\left(2 m_{2}\right) \subset O\left(2\left(m_{1}+m_{2}\right)\right)$.

LEMMA 6.5. - The $\mathbb{Z}[1 / 2]$-algebra $\mathbf{B}$ is graded commutative.

By the property of the cross-product [5], the natural diagram

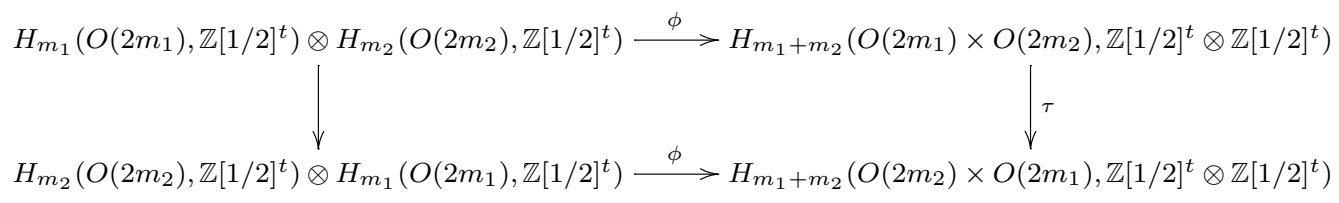

commutes up to the sign $(-1)^{m_{1} m_{2}}$.

Moreover the natural diagram

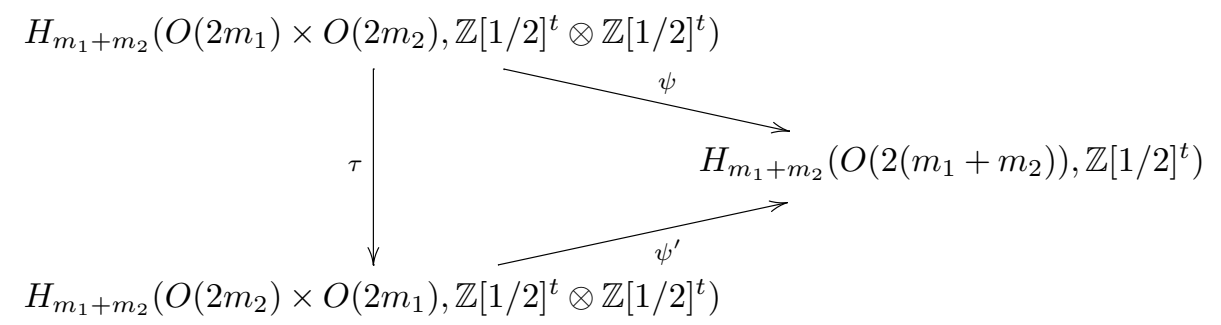

is commutative. In fact, let $g_{\sigma}$ be the matrix associated to the permutation

$$
\sigma=\left(2 m_{2}+1,2 m_{2}+2, \ldots, 2\left(m_{1}+m_{2}\right), 1,2, \ldots, 2 m_{2}\right) .
$$

Now: $\operatorname{det} g_{\sigma}=\operatorname{sign} \sigma=1$. As a result (see [5] Chap. III, Sect. 8) conjugation by $g_{\sigma}$ induces the identity on $H_{i+j}\left(O(n) \times O(m), \mathbb{Z}[1 / 2]^{t} \otimes \mathbb{Z}[1 / 2]^{t}\right)$. This implies that $\psi^{\prime} \circ \tau=\psi$.

Note that $\mathbf{B}$ is connected, and has a split augmentation $\epsilon: \mathbf{B} \rightarrow \mathbb{Z}[1 / 2]$, with augmentation ideal $\overline{\mathbf{B}}=\sum_{m \geqslant 1} H_{m}\left(O(2 m), \mathbb{Z}[1 / 2]^{t}\right)$.

Recall that a graded algebra $A=\sum_{n} A_{n}$ over a ring $R$ is quadratic if it is connected, generated as an algebra by the elements of degree 1 , and the ideal of relations is generated by some elements of degree 2. In other words, the natural morphism of $R$-algebras

$$
\mathrm{T}_{*} A_{1} \rightarrow A,
$$

ANNALES SCIENTIFIQUES DE L'ÉCOLE NORMALE SUPÉRIEURE 
where $\mathrm{T}_{*} A_{1}$ is the tensor algebra of $A_{1}$ over $R$, is surjective with kernel generated, as a two-sided ideal, by some elements of degree 2 .

THEOREM 6.6. - The $\mathbb{Z}[1 / 2]$-algebra $\mathbf{B}$ is quadratic.

This will be a consequence of the following lemma (see for example [9] Proposition 4.4, where the result is yet true for $R$ instead of $\mathbb{Q}$ ). We have written $H_{*, m}(A, R)$, for the component of weight $m$ of the Hochschild homology of $A$, with coefficients in the trivial module $R$ [41].

LEMmA 6.7. - A graded R-algebra is quadratic, if and only if, its Hochschild homology satisfies

(1) $H_{0,0}(A, R)=R, H_{0, m}(A, R)=0$, for $m \geqslant 1$.

(2) $H_{1, m}(A, R)=0$, for $m \geqslant 2$.

(3) $H_{2, m}(A, R)=0$, for $m \geqslant 3$.

Taking into account Theorem 1.4 and certain Künneth exact sequences, we get in the spectral sequence of Proposition 6.4

$E_{p, q}^{1}=0, \quad$ for $q<m$,

$E_{1, m}^{1}=H_{m}\left(O(2 m), \mathbb{Z}[1 / 2]^{t}\right)$,

$E_{2, m}^{1}=\sum_{m_{1}+m_{2}=m} H_{m_{1}}\left(O\left(2 m_{1}\right), \mathbb{Z}[1 / 2]^{t}\right) \otimes H_{m_{2}}\left(O\left(2 m_{2}\right), \mathbb{Z}[1 / 2]^{t}\right)$,

$E_{3, m}^{1}=$

$\sum_{m_{1}+m_{2}+m_{3}=m} H_{m_{1}}\left(O\left(2 m_{1}\right), \mathbb{Z}[1 / 2]^{t}\right) \otimes H_{m_{2}}\left(O\left(2 m_{2}\right), \mathbb{Z}[1 / 2]^{t}\right) \otimes H_{m_{3}}\left(O\left(2 m_{3}\right), \mathbb{Z}[1 / 2]^{t}\right)$.

Now since the abutment of the spectral sequence is 0 , in degree: $p+q<2 m$, we get the following exact sequences, where we have omitted the coefficients $\mathbb{Z}[1 / 2]^{t}$ :

(1) For $m \geqslant 2$,

$$
\sum_{m_{1}+m_{2}=m} H_{m_{1}}\left(O\left(2 m_{1}\right)\right) \otimes H_{m_{2}}\left(O\left(2 m_{2}\right)\right) \stackrel{d^{1}}{\rightarrow} H_{m}(O(2 m)) \rightarrow 0 .
$$

(2) For $m \geqslant 3$,

$$
\begin{gathered}
\sum_{m_{1}+m_{2}+m_{3}=m} H_{m_{1}}\left(O\left(2 m_{1}\right)\right) \otimes H_{m_{2}}\left(O\left(2 m_{2}\right)\right) \otimes H_{m_{3}}\left(O\left(2 m_{3}\right)\right) \\
\stackrel{d^{1}}{\rightarrow} \sum_{m_{1}^{\prime}+m_{2}^{\prime}=m} H_{m_{1}^{\prime}}\left(O\left(2 m_{1}^{\prime}\right)\right) \otimes H_{m_{2}^{\prime}}\left(O\left(2 m_{2}^{\prime}\right)\right) \stackrel{d^{1}}{\rightarrow} H_{m}(O(2 m)) \rightarrow 0 .
\end{gathered}
$$

To recognize, in these sequences, portions of the weight $m$-part of the reduced bar complex of $\mathbf{B}$, we have just to identify $d^{1}$ with the differential of Hochschild. As a direct sum of induced modules, $\mathcal{L}_{p}(n) \otimes \mathbb{Z}[1 / 2]^{t}$ is canonically (here $n=2 m$ ) isomorphic to

$$
\sum_{n_{1}+\cdots+n_{p}=n} \mathbb{Z}[1 / 2][O(n)] \otimes_{\mathbb{Z}[1 / 2]\left[O\left(n_{1}\right) \times \cdots \times O\left(n_{p}\right)\right]} \mathbb{Z}[1 / 2]^{t},
$$

by the choice of base points on the orbits given by the orthogonal decompositions of $\mathbf{k}^{n}$, associated to the partitions of the canonical basis. The Shapiro isomorphism is induced by the morphisms of pairs (see [5] Chap. III, Sect. 8, Exerc. 2)

$4^{e}$ SÉRIE - TOME $40-2007-\mathrm{N}^{\circ} 3$ 


$$
\begin{aligned}
& \left(O\left(n_{1}\right) \times \cdots \times O\left(n_{p}\right), \mathbb{Z}[1 / 2]\left[O\left(n_{1}\right) \times \cdots \times O\left(n_{p}\right)\right] \otimes_{\mathbb{Z}[1 / 2]\left[O\left(n_{1}\right) \times \cdots \times O\left(n_{p}\right)\right]} \mathbb{Z}[1 / 2]^{t}\right) \\
& \quad \rightarrow\left(O(n), \mathbb{Z}[1 / 2][O(n)] \otimes_{\mathbb{Z}[1 / 2]\left[O\left(n_{1}\right) \times \cdots \times O\left(n_{p}\right)\right]} \mathbb{Z}[1 / 2]^{t}\right) .
\end{aligned}
$$

So it suffices to observe that there are commutative diagrams of modules, where the right vertical map is the $i$-th face of $\mathcal{L}_{*}$,

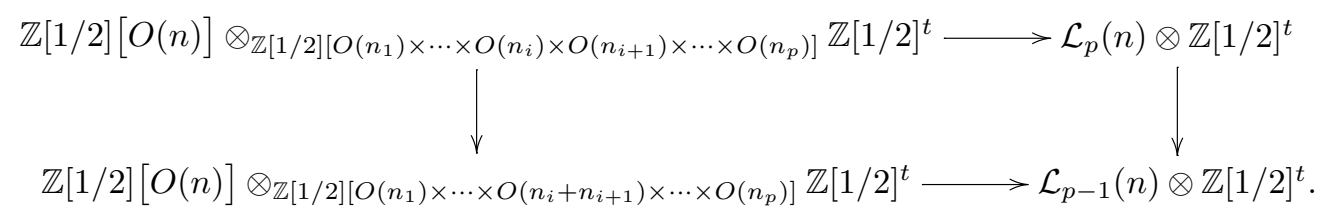

Finally, the algebra $\mathbf{B}$ satisfies the hypothesis of the lemma.

\subsection{The case of quadratically closed fields}

Henceforth, we assume that the field $\mathbf{k}$ is quadratically closed. This allows one to identify $O(2 n)$ to the orthogonal group of the hyperbolic form: $x_{1} x_{2}+x_{3} x_{4}+\cdots+x_{2 n-1} x_{2 n}$. The groups $\mathbf{k}^{\times}$and $S O(2)$ are isomorphic by the map

$$
\rho: a \mapsto\left(\begin{array}{cc}
a & 0 \\
0 & a^{-1}
\end{array}\right)
$$

We have $\mathbf{k}^{\times} \otimes \mathbb{Z}[1 / 2] \cong \mathbf{k}^{\times} / \mu_{2}(\mathbf{k})$, and

$$
H_{1}\left(O(2), \mathbb{Z}[1 / 2]^{t}\right) \cong H_{1}(S O(2), \mathbb{Z}[1 / 2]) \cong \mathbf{k}^{\times} / \mu_{2}(\mathbf{k}) .
$$

We note also that: $\mathbf{k}^{\times} / \mu_{2}(\mathbf{k}) \otimes \mathbf{k}^{\times} / \mu_{2}(\mathbf{k})=\mathbf{k}^{\times} \otimes \mathbf{k}^{\times}$.

PROPOSITION 6.8. - If $\mathbf{k}$ is quadratically closed, there is a natural isomorphism

$$
\gamma: H_{2}(S L(2, \mathbf{k}), \mathbb{Z}[1 / 2]) \rightarrow H_{2}\left(O(4), \mathbb{Z}[1 / 2]^{t}\right) .
$$

From the Hochschild-Serre spectral sequence of

$$
0 \rightarrow S O(4) \rightarrow O(4) \rightarrow\{ \pm 1\} \rightarrow 0
$$

we get an isomorphism

$$
H_{2}\left(O(4), \mathbb{Z}[1 / 2]^{t}\right) \cong H_{0}\left(\{ \pm 1\}, H_{2}(S O(4), \mathbb{Z}[1 / 2])\right) .
$$

Here the action of -1 in $H_{2}(S O(4), \mathbb{Z}[1 / 2])$ can be taken to be induced by the conjugation in $S O(4)$, by any element of $O(4)$ of determinant -1 , combined with the multiplication by -1 in $\mathbb{Z}[1 / 2]$. Let $M_{2}(\mathbf{k})$ be the space of two by two matrices. If we identify $\mathbf{k}^{4}$ with $M_{2}(\mathbf{k})$, by the map

$$
\left(x_{1}, \ldots, x_{4}\right) \mapsto\left(\begin{array}{cc}
x_{1} & -x_{3} \\
x_{4} & x_{2}
\end{array}\right),
$$

the determinant coincides with the previous hyperbolic form on $\mathbf{k}^{4}$. 
For k quadratically closed, the Spin group Spin(4) is the twofold covering

$$
\pi: S L(2, \mathbf{k}) \times S L(2, \mathbf{k}) \rightarrow S O(4),
$$

which comes from the representation of $S L(2, \mathbf{k}) \times S L(2, \mathbf{k})$ on $M_{2}(\mathbf{k})$, given by

$$
(f, g) \mapsto\left(m \mapsto f m g^{-1}\right) .
$$

An elementary calculation shows that the permutation of the two factors in $S L(2, \mathbf{k}) \times S L(2, \mathbf{k})$ is transformed by the morphism $\pi$, into the conjugation of $S O(4)$ by the matrix of determinant -1 :

$$
\left(\begin{array}{cccc}
0 & 1 & 0 & 0 \\
1 & 0 & 0 & 0 \\
0 & 0 & -1 & 0 \\
0 & 0 & 0 & -1
\end{array}\right) .
$$

This gives isomorphisms

$$
\begin{aligned}
H_{2}\left(O(4, \mathbf{k}), \mathbb{Z}[1 / 2]^{t}\right) & \cong H_{0}\left(\{ \pm 1\}, H_{2}(S O(4), \mathbb{Z}[1 / 2])\right) \\
& \cong H_{2}(S L(2, \mathbf{k}) \times S L(2, \mathbf{k}), \mathbb{Z}[1 / 2])_{\{ \pm 1\}} .
\end{aligned}
$$

Note that in the right term above, -1 acts by permutation of the two factors and by multiplication in $\mathbb{Z}[1 / 2]$.

Since $H_{1}(S L(2, \mathbf{k}), \mathbb{Z})=0$, there is no torsion problem in the Künneth exact sequence, and we get

$$
H_{2}(S L(2, \mathbf{k}) \times S L(2, \mathbf{k}), \mathbb{Z}[1 / 2]) \cong H_{2}(S L(2, \mathbf{k}), \mathbb{Z}[1 / 2]) \times H_{2}(S L(2, \mathbf{k}), \mathbb{Z}[1 / 2]) .
$$

In this isomorphism, the action of -1 becomes $(x, y) \mapsto-(y, x)$. The isomorphisms of the proposition

$$
H_{2}(S L(2, \mathbf{k}), \mathbb{Z}[1 / 2]) \stackrel{\cong}{\rightrightarrows} H_{2}\left(O(4), \mathbb{Z}[1 / 2]^{t}\right),
$$

are then given by the compositions

$$
\begin{aligned}
H_{2}(S L(2, \mathbf{k}), \mathbb{Z}[1 / 2]) & \rightarrow\left(H_{2}(S L(2, \mathbf{k}), \mathbb{Z}[1 / 2]) \times H_{2}(S L(2, \mathbf{k}), \mathbb{Z}[1 / 2])\right)_{\{ \pm 1\}} \\
& \rightarrow H_{2}\left(O(4), \mathbb{Z}[1 / 2]^{t}\right),
\end{aligned}
$$

where the first map is $x \mapsto(x,-x)$.

COROLLARY 6.9. - For a quadratically closed field $\mathbf{k}$, the following is true

$$
H_{2}\left(O(4), \mathbb{Z}[1 / 2]^{t}\right) \cong H_{2}(S L(2, \mathbf{k}), \mathbb{Z}) \cong K_{2}^{M}(\mathbf{k})
$$

This is a consequence of known results. For an infinite field (see for example [21]), the Milnor group $K_{2}^{M}(\mathbf{k})$ is isomorphic to: $H_{0}\left(\mathbf{k}^{\times}, H_{2}(S L(2, \mathbf{k}), \mathbb{Z})\right)$, where the action of $\mathbf{k}^{\times}$in $H_{2}(S L(2, \mathbf{k}), \mathbb{Z})$ stems from the extension

$$
0 \rightarrow S L(2, \mathbf{k}) \rightarrow G L(2, \mathbf{k}) \rightarrow \mathbf{k}^{\times} \rightarrow 0
$$

$4^{\mathrm{e}}$ SÉRIE - TOME $40-2007-\mathrm{N}^{\circ} 3$ 
Furthermore, when each element in $\mathbf{k}$ is a square, this action of $\mathbf{k}^{\times}$is trivial. Finally for quadratically closed fields, $K_{n}^{M}(\mathbf{k})$ is uniquely 2-divisible, for $n \geqslant 2$. In particular: $H_{2}(S L(2, \mathbf{k}), \mathbb{Z}[1 / 2])=H_{2}(S L(2, \mathbf{k}), \mathbb{Z})$.

We can be more precise. For $u, v$ two commuting elements in a group $G$, denote by $[[u, v]]$ the element of $H_{2}(G, \mathbb{Z})$ represented by the cycle $[u \mid v]-[v \mid u]$, in the bar complex. By the appendix of [16] on the Bloch-Wigner sequence ${ }^{7}$, the map

$$
\begin{aligned}
& \lambda: \mathbf{k}^{\times} \otimes \mathbf{k}^{\times} \rightarrow H_{2}(S L(2, \mathbf{k}), \mathbb{Z}), \\
& a \otimes b \mapsto[[\rho(a), \rho(b)]]
\end{aligned}
$$

is surjective with kernel generated by the Steinberg elements $a \otimes(1-a)$. Therefore it induces an isomorphism

$$
K_{2}^{M}(\mathbf{k}) \rightarrow H_{2}(S L(2, \mathbf{k}), \mathbb{Z}) .
$$

In the following result, $\theta$ is the cross product.

Proposition 6.10. - If $\mathbf{k}$ is quadratically closed, the following diagram, where the vertical maps are bijective, is commutative up to a factor 4 .

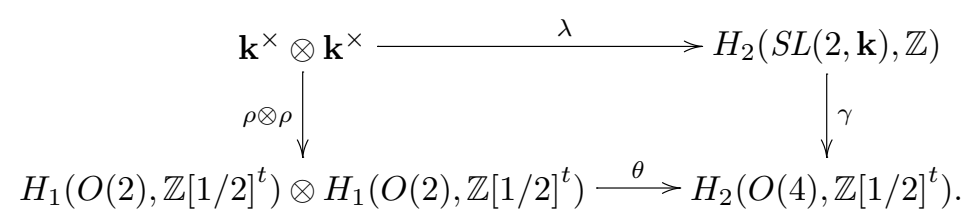

Consider the group morphism

It induces a morphism

$$
\begin{aligned}
& \nu: \mathbf{k}^{\times} \times \mathbf{k}^{\times} \rightarrow S O(4), \\
& (a, b) \mapsto\left(\begin{array}{cc}
\rho(a) & 0 \\
0 & \rho(b)
\end{array}\right) .
\end{aligned}
$$

$$
\begin{aligned}
& \nu_{*}: \wedge^{2}\left(\mathbf{k}^{\times} \times \mathbf{k}^{\times}\right) \rightarrow H_{2}\left(O(4), \mathbb{Z}[1 / 2]^{t}\right) \\
& (a, b) \wedge\left(a^{\prime}, b^{\prime}\right) \mapsto\left[\left[\nu(a, b), \nu\left(a^{\prime}, b^{\prime}\right)\right]\right] .
\end{aligned}
$$

By easy computations, we get

$$
\gamma \circ \lambda(a \otimes b)=\nu_{*}\left((a, a) \wedge(b, b)-\left(a^{-1}, a\right) \wedge\left(b^{-1}, b\right)\right),
$$

and

$$
\theta \circ(\rho \otimes \rho)(a \otimes b)=\nu_{*}((a, 1) \wedge(1, b)) .
$$

But calculating in $\wedge^{2}\left(\mathbf{k}^{\times} \times \mathbf{k}^{\times}\right)$, and using the fact that the conjugation by the permutation matrix $(13)(24)$ gives $\nu_{*}\left((a, b) \wedge\left(a^{\prime}, b^{\prime}\right)\right)=\nu_{*}\left((b, a) \wedge\left(b^{\prime}, a^{\prime}\right)\right)$, we have

$$
\begin{aligned}
\nu_{*} & \left((a, a) \wedge(b, b)-\left(a^{-1}, a\right) \wedge\left(b^{-1}, b\right)\right) \\
= & \nu_{*}((a, 1) \wedge(b, 1)+(a, 1) \wedge(1, b)+(1, a) \wedge(b, 1)+(1, a) \wedge(1, b) \\
& \left.\quad-\left(a^{-1}, 1\right) \wedge\left(b^{-1}, 1\right)-\left(a^{-1}, 1\right) \wedge(1, b)-(1, a) \wedge\left(b^{-1}, 1\right)-(1, a) \wedge(1, b)\right) \\
= & 4 \nu_{*}((a, 1) \wedge(1, b)) .
\end{aligned}
$$

\footnotetext{
${ }^{7}$ See also in this appendix the reference to [35] concerning quadratically closed fields.
} 
This proves the commutativity of the diagram.

Now, taking into account the results of this section, and the fact that the Milnor groups form a quadratic algebra, we see that the algebra $\mathbf{B}$ and the Milnor algebra $K_{*}^{M}(\mathbf{k}) \otimes \mathbb{Z}[1 / 2]$ are canonically isomorphic, for $\mathbf{k}$ quadratically closed. Theorem 1.5 is then proved.

Finally, we check that the stabilization map $H_{2}(O(2), \mathbb{Z}) \rightarrow H_{2}(O(3), \mathbb{Z})$ is not injective in general. Actually tensoring by $\mathbb{Z}[1 / 2]$, this would imply for $\mathbf{k}$ quadratically closed, the injectivity of the Steinberg map

$$
\wedge^{2} \mathbf{k}^{\times} \rightarrow K_{2}^{M}(\mathbf{k})
$$

a fact which is well known to be false in general. Therefore the range of stability in Theorem 1.1. is the best possible. The argument is as follows.

For $\mathbf{k}$ quadratically closed, let $\mathbf{k}^{3}$ be identified with the set of matrices

$$
\left(\begin{array}{cc}
x_{3} & -x_{1} \\
x_{2} & x_{3}
\end{array}\right) \in M_{2}(\mathbf{k}) .
$$

We also consider $O(3)$ as the orthogonal group of the quadratic form $x_{1} x_{2}+x_{3}^{2}$. The Spin group $\operatorname{Spin}(3)$ is then the twofold covering

$$
\Psi: S L(2, \mathbf{k}) \rightarrow S O(3),
$$

which comes from the representation of $S L(2, \mathbf{k})$ on $\mathbf{k}^{3}$, given by

$$
f \mapsto\left(m \mapsto f m f^{-1}\right) .
$$

We have

$$
\Psi\left(\left(\begin{array}{cc}
a & 0 \\
0 & a^{-1}
\end{array}\right)\right)=\left(\begin{array}{ccc}
a^{2} & 0 & 0 \\
0 & a^{-2} & 0 \\
0 & 0 & 1
\end{array}\right) .
$$

This implies that the following diagram in homology

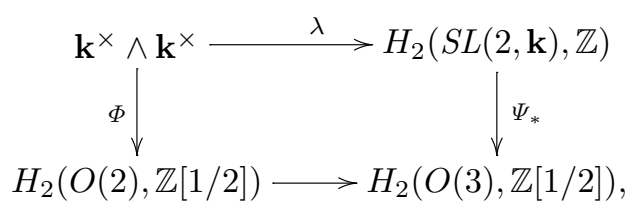

where $\Phi(a \wedge b)=[[\rho(a), \rho(b)]]$, is commutative up to a factor 4 . But one can check that, for $\mathbf{k}$ quadratically closed, the vertical maps in this diagram are isomorphisms.

\subsection{The case of the real field}

Here is a complement for $\mathbf{k}=\mathbb{R}$. Let $\sigma_{m}$ be the involution of $K_{m}^{M}(\mathbb{C}) \otimes \mathbb{Z}$, induced by the complex conjugation on $K_{m}^{M}(\mathbb{C})$ and the product by $(-1)^{m}$ on $\mathbb{Z}$. From [35], the subalgebra of $K_{*}^{M}(\mathbb{C})$ generated by $\mathbb{U}=\left\{e^{i \alpha} \in \mathbb{C}\right\}$ coincides with the direct sum of invariant subspaces

$$
\bigoplus_{m \geqslant 0}\left(K_{m}^{M}(\mathbb{C}) \otimes \mathbb{Z}\right)^{\sigma_{m}}
$$

$4^{\text {e }}$ SÉRIE - TOME $40-2007-\mathrm{N}^{\circ} 3$ 
Unfortunately we do not know if this algebra is quadratic. However we have the following.

PROPOSITION 6.11. - There is a surjective morphism of algebras

$$
\bigoplus_{m \geqslant 0} H_{m}\left(O(2 m, \mathbb{R}), \mathbb{Z}[1 / 2]^{t}\right) \rightarrow \bigoplus_{m \geqslant 0}\left(K_{m}^{M}(\mathbb{C}) \otimes \mathbb{Z}[1 / 2]\right)^{\sigma_{m}}
$$

From $[17,15]$, there is a commutative diagram, where the vertical map is bijective

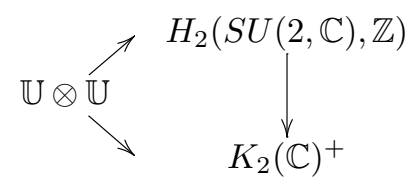

Here, $K_{2}(\mathbb{C})^{+}$is the invariant subspace under the complex conjugation.

On the other hand, proceeding as in the proofs of Propositions 6.8 and 6.10, one gets a commutative diagram

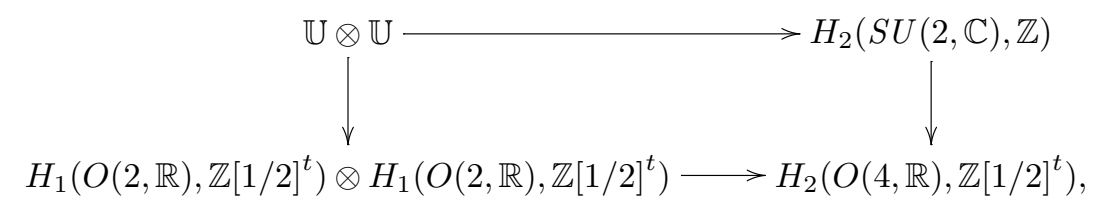

where the vertical maps are isomorphisms.

Inverting the vertical maps in the previous diagrams, the proposition follows from the fact that the algebra $\bigoplus_{m \geqslant 0} H_{m}\left(O(2 m, \mathbb{R}), \mathbb{Z}[1 / 2]^{t}\right)$ is quadratic.

\section{Acknowledgements}

I would like to thank Gaël Collinet, for pointing out the role of Steinberg modules, in relation with stability problems in homology.

\section{REFERENCES}

[1] BetLey S., Homology stability for $O(n, n)$ over a local ring, Trans. Amer. Math. Soc. 303 (1987) 413-429.

[2] Betley S., Homology stability for $O(n, n)$ over a semi-local ring, Glasgow Math. J. 32 (1990) 255259.

[3] Bökstedt M., Brun M., Dupont J., Homology of $O(n)$ and $O^{1}(1, n)$ made discrete: an application of edgewise subdivision, J. Pure Appl. Algebra 123 (1998) 131-152.

[4] Borel A., Linear Algebraic Groups, second revised ed., Grad. Texts in Math., vol. 126, SpringerVerlag, 1991.

[5] Brown K.S., Cohomology of Groups, Grad. Texts in Math., vol. 87, Springer-Verlag, 1982.

[6] Cathelineau J.-L., Homology of tangent groups considered as discrete groups and scissors congruences, J. Pure Appl. Algebra 132 (1998) 9-25.

[7] Cathelineau J.-L., Scissors congruences and the bar and cobar constructions, J. Pure Appl. Algebra 181 (2003) 141-179.

[8] Cathelineau J.-L., Projective configurations, homology of orthogonal groups and Milnor K-theory, Duke Math. J. 121 (2004) 343-387. 
[9] Cathelineau J.-L., Homology of orthogonal groups: a quadratic algebra, K-Theory 30 (2003) 1335.

[10] Cathelineau J.-L., The Pfaffian and the Lie algebra homology of skew-symmetric matrices, Math. Res. Let. 11 (2004) 315-326.

[11] Charney R.M., A generalization of a theorem of Vogtmann, J. Pure Appl. Algebra 44 (1987) 107125.

[12] Collinet G., Quelques propriétés homologiques du groupe $O_{n}(\mathbb{Z}[1 / 2])$, Thèse, Paris, 2002.

[13] Dieudonné J., Sur les groupes classiques, Hermann, Paris, 1958.

[14] Dupont J.L., Algebras of polytopes and homology of flag complexes, Osaka J. Math. 19 (1982) 599-641.

[15] Dupont J.L., Scissors Congruences, Group Homology and Characteristic Classes, Nankai Tracts in Mathematics, vol. 1, World Scientific, 2001.

[16] Dupont J.L., SAH C.H., Scissors congruences II, J. Pure Appl. Algebra 25 (1982) 159-195.

[17] Dupont J.L., PARrY W., SAH C.H., Homology of classical Lie groups made discrete II, J. Algebra 113 (1988) 215-260.

[18] FriedLANDER E.M., Homology stability for classical groups over finite fields, in: Lecture Notes in Math., vol. 551, Springer-Verlag, 1976, pp. 290-302.

[19] GuIN D., Homologie du groupe linéaire et $K$-théorie de Milnor des anneaux, J. Algebra 123 (1989) 27-59.

[20] Goncharov A., Volumes of hyperbolic manifolds and mixed Tate motives, J. Amer Math. Soc. 12 (1999) 569-618.

[21] Hutchinson K., A new approach to Matsumoto's theorem, K-Theory 4 (1990) 181-200.

[22] VAN DER KALLEN W., Homology stability for linear groups, Invent. Math. 60 (1980) 269-295.

[23] KARouBi M., Théorie de Quillen et homologie du groupe orthogonal, Ann. of Math. 112 (1980) 207257.

[24] Karoubi M., Le théorème fondamental de la K-théorie hermitienne, Ann. of Math. 112 (1980) 259282.

[25] LAM T.Y., The Algebraic Theory of Quadratic Forms, Benjamin, 1973.

[26] LodAy J.L., Procesi C., Homology of symplectic and orthogonal algebras, Adv. in Math. 69 (1988) 93-108.

[27] Milnor J., Algebraic K-theory of quadratic forms, Invent. Math. 9 (1970) 318-344.

[28] MirzaiI B., VAn Der Kallen W., Homology stability for unitary groups, Doc. Math. 7 (2002) 143-166.

[29] Nesterenko T.Y., SuSLin A.A., Homology of the full linear group over a local ring and Milnor's K-theory, Math. SSSR Izvestija 34 (1990) 121-145.

[30] PANIN I., Homological stabilization for the orthogonal and symplectic groups, J. Soviet Math. 52 (1990) 3165-3170.

[31] PANIN I., On stabilization for orthogonal and symplectic algebraic K-theory, Leningrad Math. J. 1 (1990) 741-764.

[32] Rosenberg J., Algebraic $K$-Theory and Its Applications, Grad. Text Math., vol. 147, SpringerVerlag, 1994.

[33] SAH C.H., Homology of classical Lie groups made discrete, I. Stability theorems and Schur multipliers, Comment. Math. Helv. 61 (1986) 308-347.

[34] SAH C.H., Hilbert's Third Problem: Scissors Congruence, Research Notes in Math., vol. 33, Pitman, 1979.

[35] SAH C.H., WAgOner J.B., Second homology of Lie groups made discrete, Comm. Algebra 5 (1977) 611-642.

[36] SusLin A.A., Homology of $G L_{n}$, characteristic classes and Milnor $K$-theory, in: Lecture Notes in Math., vol. 1046, Springer-Verlag, 1984, pp. 357-375.

[37] SusLin A.A., Stability in algebraic $K$-theory, Oberwolfach, 1980, in: Lecture Notes in Math., vol. 966, Springer-Verlag, 1982, pp. 303-333.

[38] Vogtman K., Homology stability for $O_{n, n}$, Comm. Algebra 7 (1979) 9-38.

[39] Vogtman K., Spherical posets and homology stability for $O_{n, n}$, Topology 20 (1981) 119-132.

4e SÉRIE - TOME $40-2007-\mathrm{N}^{\circ} 3$ 
[40] Vogtman K., A Stiefel complex for the orthogonal group of a field, Comment. Math. Helv. 57 (1982) $11-21$.

[41] Weibel C., An Introduction to Homological Algebra, Cambridge Studies in Advanced Mathematics, vol. 38, Cambridge Univ. Press, 1994.

(Manuscrit reçu le 20 septembre 2006; accepté le $1^{\text {er }}$ mars 2007.)

\footnotetext{
Jean-Louis CATHELINEAU Université de Nice - Sophia-Antipolis,

Laboratoire J.-A. Dieudonné, UMR CNRS 6621, Parc Valrose,

06108 Nice Cedex 02, France

E-mail: cathe@math.unice.fr
} 